\title{
A novel treatment of cystic fibrosis acting on-target: cysteamine plus epigallocatechin gallate for the autophagy-dependent rescue of class II-mutated CFTR
}

\author{
A Tosco ${ }^{1}$, F De Gregorio ${ }^{1}$, S Esposito², D De Stefano ${ }^{2}$, I Sana ${ }^{2}$, E Ferrari ${ }^{2}$, A Sepe ${ }^{1}$, L Salvadori ${ }^{1}$, P Buonpensiero ${ }^{1}$, A Di Pasqua ${ }^{1}$, \\ R Grassia ${ }^{3}$, CA Leone ${ }^{3}$, S Guido ${ }^{4}$, G De Rosa ${ }^{5}$, S Lusa ${ }^{6}$, G Bona ${ }^{7}$, G Stoll ${ }^{8,9,10,11}$, MC Maiuri ${ }^{9}$, A Mehta ${ }^{12}$, G Kroemer ${ }^{\star, 9,13,14,15,16,17}$, \\ L Maiuri ${ }^{*, 2,7}$ and V Raia ${ }^{*, 1}$
}

\begin{abstract}
We previously reported that the combination of two safe proteostasis regulators, cysteamine and epigallocatechin gallate (EGCG), can be used to improve deficient expression of the cystic fibrosis transmembrane conductance regulator (CFTR) in patients homozygous for the CFTR Phe508del mutation. Here we provide the proof-of-concept that this combination treatment restored CFTR function and reduced lung inflammation $(P<0.001)$ in Phe508del/Phe508del or Phe508del/null-Cftr (but not in Cftr-null mice), provided that such mice were autophagy-competent. Primary nasal cells from patients bearing different class II CFTR mutations, either in homozygous or compound heterozygous form, responded to the treatment in vitro. We assessed individual responses to cysteamine plus EGCG in a single-centre, open-label phase-2 trial. The combination treatment decreased sweat chloride from baseline, increased both CFTR protein and function in nasal cells, restored autophagy in such cells, decreased CXCL8 and TNF- $\alpha$ in the sputum, and tended to improve respiratory function. These positive effects were particularly strong in patients carrying Phe508del CFTR mutations in homozygosity or heterozygosity. However, a fraction of patients bearing other CFTR mutations failed to respond to therapy. Importantly, the same patients whose primary nasal brushed cells did not respond to cysteamine plus EGCG in vitro also exhibited deficient therapeutic responses in vivo. Altogether, these results suggest that the combination treatment of cysteamine plus EGCG acts 'on-target' because it can only rescue CFTR function when autophagy is functional (in mice) and improves CFTR function when a rescuable protein is expressed (in mice and men). These results should spur the further clinical development of the combination treatment.
\end{abstract}

Cell Death and Differentiation (2016) 23, 1380-1393; doi:10.1038/cdd.2016.22; published online 1 April 2016

Cystic fibrosis (CF), the most common lethal recessive disease in Caucasians, affects $\sim 70000$ subjects worldwide and results from mutations in the cystic fibrosis transmembrane conductance regulator (CFTR). ${ }^{1,2}$ Mutation-specific CFTR-repairing therapies with a channel-potentiator (Ivacaftor) are available for $\sim 5 \%$ of CF patients bearing membraneresident class III mutants. ${ }^{3}$ In contrast, for the vast majority of CF patients bearing the most common F508del-CFTR mutation, an FDA-approved combined treatment with the corrector Lumacaftor (that promotes ER to plasma membrane (PM) traffic) and Ivacaftor is marginally effective. ${ }^{4-7}$ F508delCFTR is retained in the endoplasmic reticulum (ER) and prematurely degraded before it reaches the $\mathrm{PM}^{8}{ }^{8}$ Besides a gating defect, ${ }^{8}$ F508del is unstable and rapidly dismissed from the PM even upon treatment with correctors. When Ivacaftor is combined in vitro with CFTR correctors, the former counteracts the latter by further decreasing F508del-CFTR PM stability after rescue. ${ }^{9,10}$ This might explain the marginal clinical effects of this combination in F508del-CFTR patients ${ }^{4}$ as it fails to reverse sweat test, a surrogate marker of disease reversal, ${ }^{11,12}$ consistent with putative 'off-target' effects.

Discordance in therapeutic response rate complicates mutation-specific approaches. Existing candidate drugs, as Lumacaftor, fail to act on other class II mutants, ${ }^{13,14}$ show poor

${ }^{1}$ Regional Cystic Fibrosis Center, Pediatric Unit, Department of Translational Medical Sciences, Federico II University, Naples 80131 , Italy; ${ }^{2}$ European Institute for Research in Cystic Fibrosis, Division of Genetics and Cell Biology, San Raffaele Scientific Institute, Milan 20132, Italy; ${ }^{3}$ Otorhinolaryngology Unit, Monaldi Hospital, Naples 80131 , Italy; ${ }^{4}$ Department of Chemical, Materials and Production Engineering; Federico II University, Naples, Italy; ${ }^{5}$ Department of Pharmacy, School of Pharmacy, Federico II University, Naples 80131, Italy; ${ }^{6}$ Department of Biochemistry, Biophysics and General Pathology, Second University of Naples, Naples 80138 , Italy; ${ }^{7}$ SCDU of Pediatrics, Department of Health Sciences, University of Piemonte Orientale, Novara 28100, Italy; ${ }^{8}$ Université Paris Descartes, Sorbonne Paris Cité, Paris, France; ${ }^{9}$ Equipe 11 labellisée Ligue Nationale contre le Cancer, Centre de Recherche des Cordeliers, Paris, France; ${ }^{10}$ Institut National de la Santé et de la Recherche Médicale, Paris, France; ${ }^{11}$ Université Pierre et Marie Curie, Paris, France; ${ }^{12}$ Division of Cardiovascular and Diabetes Medicine, Ninewells Hospital and Medical School, University of Dundee, Dundee, UK; ${ }^{13}$ INSERM U1138, Centre de Recherche des Cordeliers, Paris, France; ${ }^{14}$ Université Paris Descartes, Paris, France; ${ }^{15}$ Metabolomics and Cell Biology Platforms, Institut Gustave Roussy, Villejuif, France; ${ }^{16}$ Pôle de Biologie, Hôpital Européen Georges Pompidou, AP-HP, Paris, France and ${ }^{17}$ Karolinska Institute, Department of Women's and Children's Health, Karolinska University Hospital, Stockholm 17176, Sweden

${ }^{*}$ Corresponding author: G Kroemer or L Maiuri or V Raia, European Institute for Research in Cystic Fibrosis, Division of Genetics and Cell Biology, San Raffaele Scientific Institute, Milan 20132, Italy. Tel: +39 3207981514; E-mail: kroemer@orange.fr or maiuri.luigi@hsr.it or raia@unina.it

Abbreviations: CF, cystic fibrosis; CFTR, cystic fibrosis transmembrane conductance regulator; EGCG, epigallocatechin gallate; ER, endoplasmic reticulum; PM, plasma membrane; TG2, tissue transglutaminase; BECN1/Beclin 1, autophagy-related protein Beclin 1; SQSTM1, sequestosome 1; CSNK2, casein kinase 2; KO, knockout; RPD, rectal potential difference; CXCL2, chemochine (C-X-C motif) ligand 2; TNF, tumor necrosis factor; WT, wild type; CXCL8, chemokine (C-X-C motif) ligand 8; FEV 1 , forced expiratory volume at first second

Received 25.11.15; revised 25.1.16; accepted 27.1.16; Edited by M Piacentini; published online 01.4.16 
efficacy in vitro and lack of benefit in vivo ${ }^{5}$ in CF patients bearing only one copy of F508del. This indicates that a different rationale is needed based on mechanistic rather than mutation-specific approaches, and suggests strategies focusing on individual and not average responses to therapy. ${ }^{13}$

Recent results suggest that CFTR orchestrates a proteostatic network that influences multiple cellular functions by acting as a hub protein. ${ }^{15}$ This hub-dysfunction model proposes that the proteostasis network is widely deranged, both in transgenic CF mice and in primary epithelial cells from F508del-CFTR homozygous patients, at two levels. First, autophagy, the major mechanism determining cytoplasmic protein turnover, is blocked owing to tissue transglutaminase (TG2)-mediated depletion of the essential autophagy-related protein Beclin 1 (BECN1), leading to secondary accumulation of the autophagic substrate SQSTM1/p62. ${ }^{16}$ Second, peptide fragments released from proteolytically cleaved F508delCFTR provoke an overactivation of the pleiotropic protein kinase CK2, which in turn contributes to F508del-CFTR degradation. ${ }^{17}$ Combined inhibition of both TG2 by the repurposed cysteamine, FDA-approved for the treatment of cystinosis, ${ }^{18,19}$ (which reestablishes autophagy) and overactive CK2 by the over-the-counter green-tea flavonoid epigallocatechin gallate (EGCG) synergize in vitro and thereby rescue and stabilize, respectively, a functional F508del-CFTR protein at the PM, both in mice and in primary nasal cells from F508del-CFTR homozygotes. ${ }^{20}$ This prompted a pilot trial combining cysteamine and EGCG in ten F508del-CFTR homozygotes, showing that the combination treatment was well tolerated, reverted sweat chloride toward normal and significantly attenuated biomarkers of airway inflammation. ${ }^{20}$

Here, we provide new insights from newly generated transgenic mice and patients primary cells and investigate in a phase-2 clinical trial the individual patient responsiveness to a combination of cysteamine plus EGCG in CF patients bearing different classes of CFTR mutation. Moreover, we evaluate the feasibility of using both functional and mechanistic biomarkers as a prediction test of patient responsiveness to facilitate a future personalized approach to precision CF therapy.

\section{Results}

Proof-of-concept studies in mice. To provide the proof-ofconcept for the use of cysteamine plus EGCG in CF patients bearing one copy of F508del-CFTR and another severe CFTR mutation with negligible residual CFTR activity, F508del-CFTR homozygous mice (Cftr ${ }^{\text {F508del/F508del }}$ ) were backcrossed with transgenic knockout (KO) Cftr mice $\left(\mathrm{Cttr}^{-1-}\right)$ to obtain F508del/null CFTR heterozygous mice ( $\mathrm{Cftr}{ }^{\mathrm{F} 508 \mathrm{dell}-}$ ). Mice were gavaged with cysteamine or EGCG alone or cysteamine plus EGCG for 5 days followed by EGCG alone or vehicle for further 2 weeks. ${ }^{20}$ The primary end point of this mouse trial (Supplementary Figure S1) was the rescue of CFTR function and protein. Secondary end points were the restoration of $\mathrm{BECN1} 1^{20-22}$ and the reduction of $\mathrm{CxCl} 2$ (chemokine[C-X-C motif]-ligand 2) and Tnf-a (tumor necrosis factor) transcripts in lung homogenates.
Cysteamine alone or combined with EGCG (but not EGCG alone) restored rectal potential difference (RPD) responses to forskolin in vivo to mean $79.4 \%$ (95\% confidence interval $(\mathrm{Cl})$ : 68.1-90.8\%) of WT controls in $\mathrm{Cftr}^{\text {F508del/F508del mice and }}$

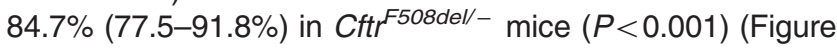
$1 \mathrm{a}$ and $\mathrm{b})$. The treatment significantly improved the forskolininduced increase in short-circuit current $\left(I_{\mathrm{sc}}\right)$ in mouse ileum mounted in Ussing chambers to $130 \%(123.6-136.4 \%)$ and $138.7 \%$, (130.4-147.0\%) of WT controls $(P<0.001)$, respectively, which was partially reverted by a selective CFTR

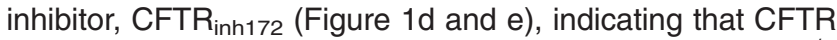
function was restored by the treatment. The inability of $\mathrm{Cftr}^{-1-}$ mice to mount a similar response to cysteamine (Figure 1c and $f$ ) indicated that mutated CFTR protein is indispensable for therapeutic effect. No effects of cysteamine or EGCG on CFTR function were observed in WT mice (Figure $1 \mathrm{~h}$ and Supplementary Figure S2). Cysteamine alone or combined with EGCG, but not EGCG alone, rescued mature CFTR protein (band C) expression to $>50 \%$ of WT values and restored $\mathrm{BECN1}$ expression in intestinal homogenates from

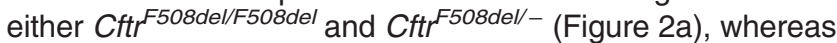
no CFTR protein was detected in intestine from $\mathrm{Cftr}^{-/}$mice, regardless of treatment (Figure $2 b$ ).

To determine whether the effects of cysteamine on F508delCFTR rescue were linked to its capacity to restore autophagy,

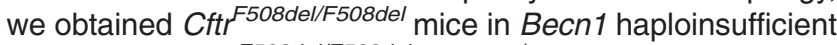

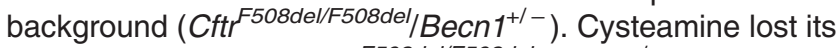
therapeutic efficacy in $\mathrm{Cftr}^{\mathrm{F} 508 \mathrm{del} / \mathrm{FF} 08 \mathrm{del}} \mathrm{BeCn}^{+/-}$mice (Figure $1 \mathrm{~g}-\mathrm{i}$ and Figure $2 \mathrm{c}$ ), supporting the importance of BECN1dependent autophagy for drug effects.

The combination treatment significantly reduced $\mathrm{Cxcl} 2$ and Tnf-a mRNA levels in lung homogenates from Cftr F508del/F508del and CftrF08del/- mice (Figure 2d). All the responses observed in these mice were maintained for 2 weeks beyond cysteamine withdrawal, provided that EGCG treatment was maintained during this period $(P<0.001$ versus mice treated with vehicle during cysteamine withdrawal) (Figures 1 and 2).

Taken together, proof-of-concept data supported the feasibility of treating autophagy-competent mice expressing at least one F508del-CFTR allele by a combination of cysteamine and EGCG.

Human studies. To translate preclinical data in humans, 52 consenting CF patients were sequentially enrolled at Regional Cystic Fibrosis Care Center, University of Naples Federico II, in a phase-2 clinical trial. Patients were F508del homozygous or compound heterozygous for (i) F508del or another class II mutation on one allele and a severe CFTR mutation with minimal residual CFTR activity (class I or II) on the other allele or (ii) class I mutations on both alleles (negligible CFTR synthesized). Schedule of treatment comprised oral cysteamine alone for 8 weeks, followed by cysteamine plus EGCG for further 4 weeks (or 8 weeks in a subgroup of 10 F508del-CFTR homozygous patients) followed by EGCG alone for additional 8 weeks (Figure 3). Throughout, all subjects continued their pre-study medication. Forty-two of 52 subjects who accepted the treatment schedule were assigned to three different cohorts by genotype, whereas 10 who declined treatment schedule, all bearing F508del or other class II CFTR mutations, were 
allocated to the untreated 'standard-of-care' control group (cohort 4) to follow the natural history of disease during an overlapping period. Baseline characteristics of study population and scheduled visits are reported in Supplementary Tables S1-S5.

In vitro studies before in vivo treatment: Before in vivo treatment, primary nasal epithelial cells freshly collected by nasal brushing at baseline were challenged in vitro with cysteamine and/or EGCG, and then kept in culture with medium alone or EGCG up to $48 \mathrm{~h} .^{20}$ The combination treatment in vitro restored both CFTR function and CFTR band $\mathrm{C}$ protein in $\mathrm{PM}$ fractions up to $>50 \%$ of non-CF controls in all but one patient bearing at least one class II mutation. F508del homozygous patients were responsive, as described, ${ }^{20}$ whereas all subjects bearing two class I mutations were unresponsive. The effects of combination treatment in vitro in class II patients persisted up to $48 \mathrm{~h}$ following cysteamine washout, provided that EGCG was maintained in the culture medium (Figure 4, Supplementary Table S6).
In vivo treatment: In total, $88.5 \%$ of patients completed the study. Mean adherence to treatment was $93.8 \%$. Given the short half-life of cysteamine, data from two patients with adherence $<60 \%$ were excluded. The pharmacokinetic profile of cysteamine was as described in our pilot trial (Supplementary Figure S3). ${ }^{20}$ The incidence of adverse events was comparable to that observed in cysteaminetreated cystinosis (Supplementary Tables S7-S11).

Treatment efficacy. The primary end point was the change in CFTR function, measured by a two-site assessment: decrease of sweat chloride values from baseline of $>15 \%$ coupled to increase of CFTR function and CFTR band C protein in nasal epithelial cells of $>15 \%$ of non-CF controls. Secondary end points were (i) restoration of autophagy in nasal brushing (mechanistic end point biomarkers); (ii) reduction from baseline of inflammatory cytokines in both nasal cells and sputum (biomarker of organ inflammation); (iii) absolute change from baseline in percentage of predicted forced expiratory volume at $1 \mathrm{~s}\left(\mathrm{ppFEV}_{1}\right)$ (marker of clinical efficacy).
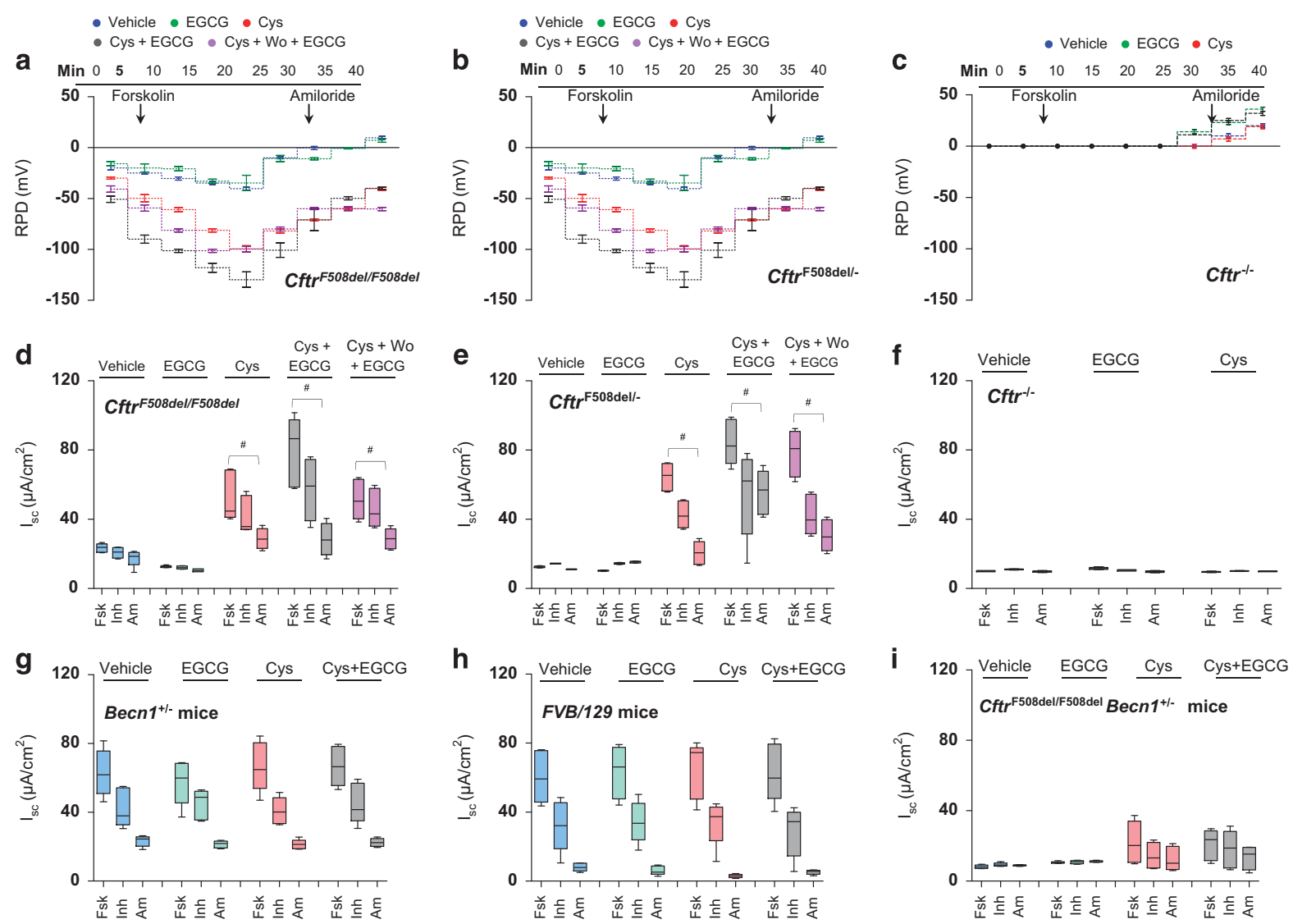

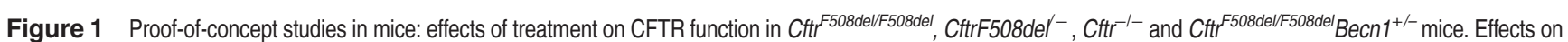
CFTR function of oral administration of different combinations of cysteamine and EGCG in Cftr ${ }^{F 508 d e l / F 508 d e l}$ (a and d d), $\mathrm{Cftr}^{\mathrm{F}}{ }^{-58 \mathrm{del} /}-(\mathbf{b}$ and $\mathbf{e})$ and $\mathrm{Cftr}^{-1-}$ (c and f) mice. The different treatment schedules are indicated. (a-c) Rectal potential difference (RPD) in vivo in response to $20 \mu \mathrm{M}$ forskolin (Fsk) (mean \pm S.D.); (d-f) CFTR-dependent Cl ${ }^{-}$ secretion measured by means of forskolin-induced increase of the chloride current $\left(\mathrm{I}_{\mathrm{sc}}\left(\mu \mathrm{A} / \mathrm{cm}^{2}\right)\right)$ ex vivo in the ileum mounted in Ussing chambers and effects of CFTR inhibition

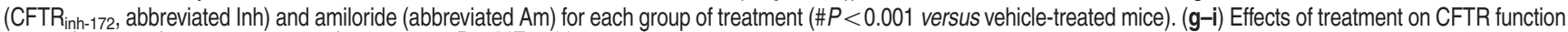

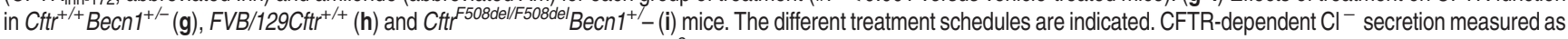
by means of forskolin-induced increase of the chloride current $\left(\mathrm{I}_{\mathrm{sc}}\left(\mu \mathrm{A} / \mathrm{cm}^{2}\right)\right)$ ex vivo in the ileum mounted in Ussing chambers and effects of CFTR inhibition (CFTR $\mathrm{inh}_{172}$, abbreviated $\mathrm{Inh}$ ) and amiloride (abbreviated Am) for each group of treatment $\left({ }^{\#} P<0.001\right.$ versus vehicle-treated mice). Eight-week-old mice, $n=10$ per group of treatment. Treatments: Cys, cysteamine; EGCG, epigallocatechin gallate; Cys+EGCG, 5 days treatment with cysteamine and EGCG; Cys+Wo+EGCG, 5 days treatment with cysteamine and EGCG followed by 2 weeks of cysteamine washout in the presence of EGCG 
a

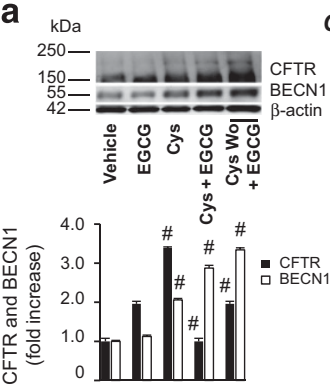

C

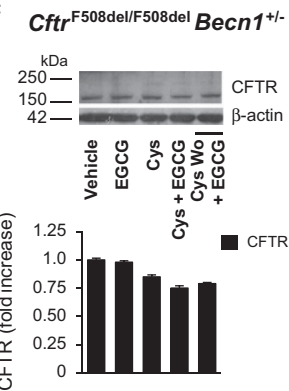

Cftr ${ }^{\text {F508del/- }}$

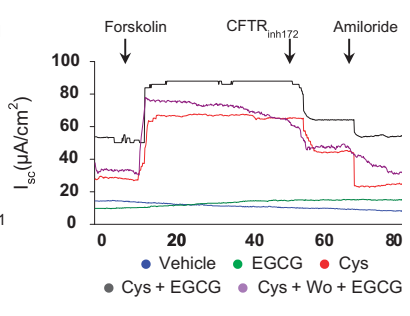

b

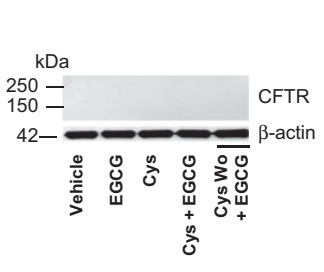

Cftr-

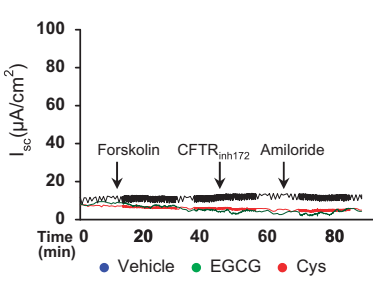

d

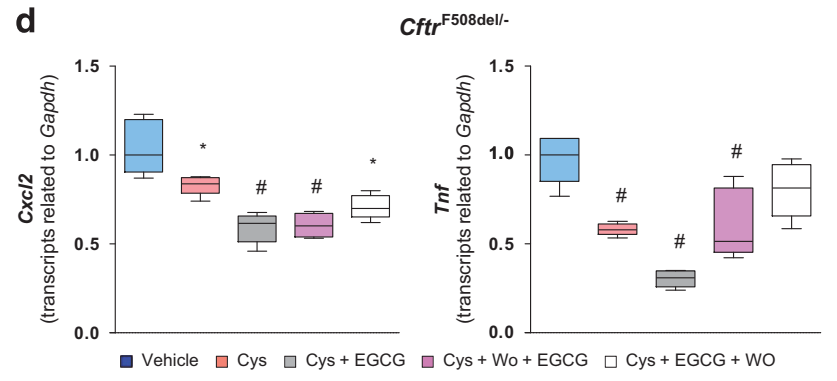

Figure 2 Proof-of concept studies in mice: effects of treatment on rescue of functional CFTR protein and lung inflammation in CftrF508del- mice. (a) Representative immunoblot with anti-CFTR (clone CF3 Abcam) and BECN1 (clone Ab55878) (top leff) and densitometric measurement as fold increase respect to vehicle-treated mouse normalized to $\beta$-actin levels (bottom left) in one $\mathrm{Cftr}^{\mathrm{F}}{ }^{208 \mathrm{del} / \mathrm{C}}$ - mouse per treatment group; (right) assessment of CFTR-dependent $\mathrm{Cl}^{-}$secretion measured by means of forskolininduced increase of the chloride current $\left(\mathrm{I}_{\mathrm{sc}}\left(\mu \mathrm{A} / \mathrm{cm}^{2}\right)\right)$ ex vivo in the ileum mounted in Ussing chambers and effects of CFTR inhibition (CFTR inh-172, abbreviated Inh) and amiloride (abbreviated Am) (right) in one mouse per treatment group. (b) Representative immunoblot with anti-CFTR (clone CF3 Abcam) (left) and assessment of CFTRdependent $\mathrm{Cl}^{-}$secretion (right) in one $\mathrm{Cftr}^{-1}$ mouse per treatment group. (c) Representative immunoblot with anti-CFTR (clone CF3 Abcam) (top) and densitometric measurement (bottom) in one Cftr ${ }^{F 508 d e l / F 508 d e l} B e c n 1^{+/-}$mouse per treatment group mean \pm S.D. of independent measurements; ${ }^{\#} P<0.001$ versus vehicle-treated mice (ANOVA). (d) Effects of oral administration of cysteamine and EGCG on cytokine expression in lung homogenates from Cftr ${ }^{\text {F508del/ }-}$ mice. The different treatment schedules are indicated. Cxcl2 (leff) and Tnf (right) mRNA levels in lung homogenates from 8-week-old mice ( $n=10$ per group of treatment). $\left({ }^{\star} P<0.01,{ }^{\#} P<0.001\right.$ versus untreated mice). Cys, cysteamine; EGCG, epigallocatechin gallate; Cys+EGCG, 5 days treatment with cysteamine and EGCG; Cys+Wo+EGCG, 5 days treatment with cysteamine and EGCG followed by 2 weeks of cysteamine washout in the presence of EGCG; Cys+EGCG+Wo, 5 days treatment with cysteamine and EGCG followed by 2 weeks of washout of both cysteamine and EGCG

The study met the primary end point of efficacy. In the 34 treated patients who completed the study the combined therapy reduced sweat chloride concentrations by mean $-18.0 \mathrm{mmol} /$ liter (range, -66 to $21 ; 95 \% \mathrm{Cl},-23.7$, $-12.3 \mathrm{mmol} / \mathrm{l})$, corresponding to mean $-19.8 \%$ ( -50 to $24.4 \% ; 95 \% \mathrm{Cl},-25.5 ;-14.1 \%)$ as compared with baseline $(P<0.0001, t$-test treatment versus baseline) (Figure $5 \mathrm{a}$, Supplementary Figure S4) and increased CFTR function in nasal cells from mean $(95 \% \mathrm{Cl}) 5.8 \%(4.8,6.7 \%)$ at week 0 up to mean $20.9 \%(18.7,23.1 \%)$ (expressed as percentage of non-CF controls) after combination treatment $(P<0.0001$ versus baseline) (Figure $5 \mathrm{~b}$ ). Accordingly, the abundance of band C CFTR protein increased from mean $9.9 \%(95 \% \mathrm{Cl}$, $9.4,10.5 \%)$ of non-CF control values to $60.5 \%(55.4,65.7 \%)$ after combined treatment $(P<0.0001$ versus baseline $)$ (Figure 5c,Supplementary Figure S5a). The increase percentages of CFTR function and band $\mathrm{C}$ protein in nasal brushing are strongly correlated (Pearson's correlation coefficient $=0.61$ with the associate $P=0.0001$ ). Notably, the percentage change of sweat chloride is negatively correlated with change in CFTR function in nasal cells (Pearson's correlation coefficient $=-0.47$ with the associated $P=0.005$, Spearman's correlation coefficient $=-0.44$ with $P=0.009$ ) (Figure $5 \mathrm{~d}$ ). Changes of primary end points were negligible in the untreated cohort 4 throughout the study $(P>0.5$ versus baseline) (Figure $5 a-c)$.

Regarding the secondary end points, the combination treatment increased BECN1 levels in nasal cells from mean $(95 \% \mathrm{Cl}) 22.4 \%(21.9,22.8 \%)$ of non-CF controls at the baseline, up to $53.1 \%(49.6,56.6 \%)$ and decreased SQSTM1/ p62 from mean $276.3 \%(274.1,278.6 \%)$ of controls to $134.0 \%$ $(119.5,148.6 \%)(P<0.0001$ versus baseline) (Supplementary Figure S5b). Moreover, in treated patients, CXCL8 (chemokine [C-X-C motif]-ligand 8) and TNF- $a$ mRNA levels in nasal brushing and protein levels in the sputum significantly decreased after combination treatment respect to baseline $(P<0.001)$ with a mean percentage $(95 \% \mathrm{Cl})$ decrease of CXCL8 and TNF- $a$ of $-57.7 \%(-45.5,-69.9 \%)$ and $-55.8 \%$ $(-45.5,-66.2 \%)$, respectively, in nasal cells and $-45.6 \%$ $(-34.5,-56.7 \%)$ and $-26.3 \%(-14.8,-37.9 \%)$ in the sputum. Notably, cytokine expression remained attenuated after 4 weeks following drug discontinuation (Figure 6a, $d$ and e,Supplementary Figure S6). Changes in either autophagy markers or cytokine expression (Figure 6a) were negligible in the untreated cohort 4 throughout the study.

In this short-term clinical trial (the effective combination treatment covers 4 or 8 weeks of the 24-week study) a mean absolute change from baseline, although not significant, of 3.2 


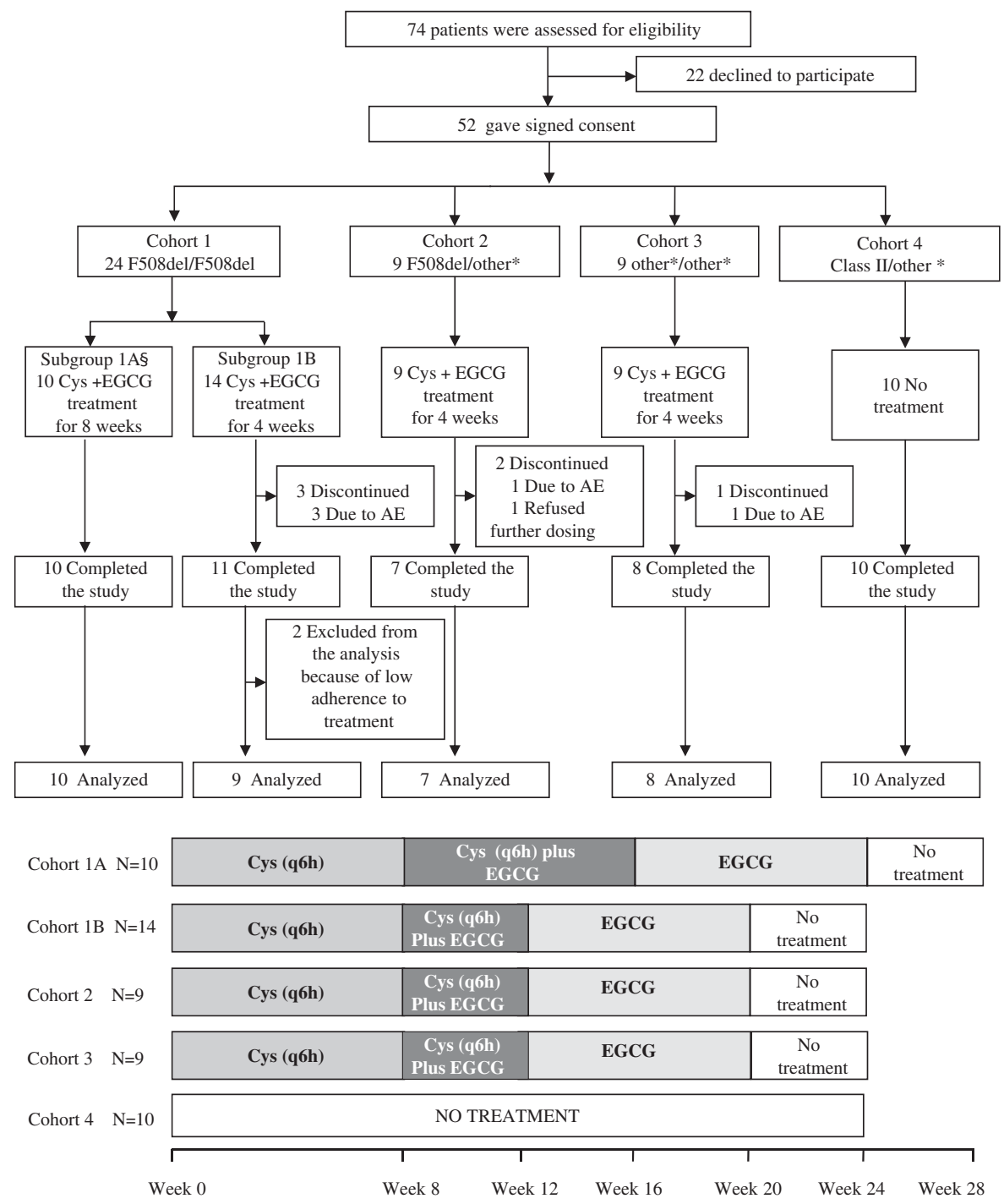

Figure 3 Study profile and study design. Other ${ }^{*}=$ Class I or II mutations. § Patients (Subgroup $1 \mathrm{~A}, N=10$ ) had already participated to the first part of the study and were enrolled after at least 4 months of washout when all laboratory and clinical parameters returned at the values registered prior study. Dosage: cysteamine: $<12$ years: $900 \mathrm{mg} / \mathrm{die}$ in four doses; > 12 years: $1200 \mathrm{mg} /$ die in four doses. EGCG: $270 \mathrm{mg} /$ die in one dose. AE, adverse events; Cys, cysteamine; EGCG, epigallocathecin gallate

in ppFEV $1(95 \% \mathrm{Cl}, 0.2,6.3)$ was observed after combination treatment and tended to increase at the post-treatment visit (Figure 6b). The increase was greater $(6.2(95 \% \mathrm{Cl}, 1.0,11.4))$ in the subgroup of patients (subgroup $A$ of cohort 1) who received combination treatment for 8 weeks (Figure 6c). By contrast, negligible mean changes of $-0.7(95 \% \mathrm{Cl},-4.7,2.8)$ were observed in the untreated group (Figure 6b).

Individual responsiveness to treatment. Given the wellknown inter- and intra-individual variability of either ppFEV $_{1}^{23}$ or sweat chloride concentrations, ${ }^{24}$ and the discordance in response rate to treatment even in patients with the same genotype ${ }^{25,26}$ we assessed the individual patient responsiveness to treatment by analyzing the proportion of patients who met primary end point criteria. We pre-specified that two criteria must be simultaneously fulfilled to define responsiveness: (1) decrease of sweat chloride concentrations to values below $-99 \% \mathrm{Cl}$ from mean individual values during the preceding 24 months, including week -0 value; 2) increase of both CFTR function and band $\mathrm{C}$ protein in nasal cells to $>15 \%$ of non-CF controls. Next, we analyzed the proportion of participants who had fulfilled the primary end point (referred to as responders), who also show: (i) autophagy rescue in nasal cells (BECN1 $>50 \%$ of nonCF controls and SQSTM1/p62 levels $<50 \%$ from baseline); (ii) decrease of CXCL8 and/or TNF-a levels in nasal cells and/or sputum by $>30 \%$ from baseline; (iii) absolute change from baseline in $\mathrm{ppFEV}_{1}$. Individual values are shown in Figure 5e, and Figures 6d,e and 7, Supplementary Figure S7 and Supplementary Tables S12-13.

Among 34 treated participants, 26 (76.5\%) fulfilled composite primary end point criteria after combination treatment. Sweat chloride fell below $60 \mathrm{mmol} / \mathrm{liter}$ in eight subjects bearing at least one class II mutation (Figure 5e). Patients bearing class I mutations on both alleles were non-responders. None of untreated participants met the 


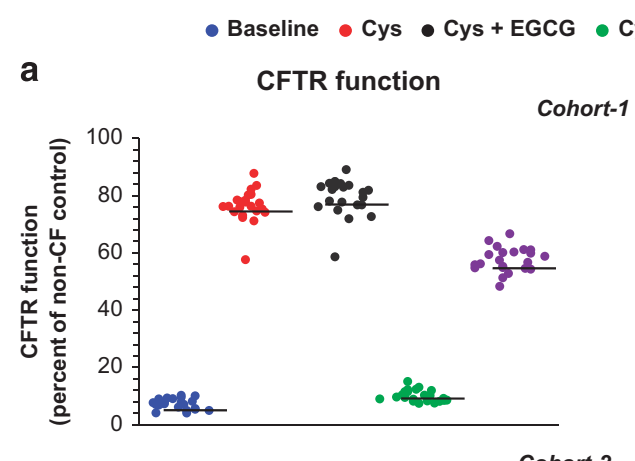

+ EGCG + Wo Cys + Wo + EGCG

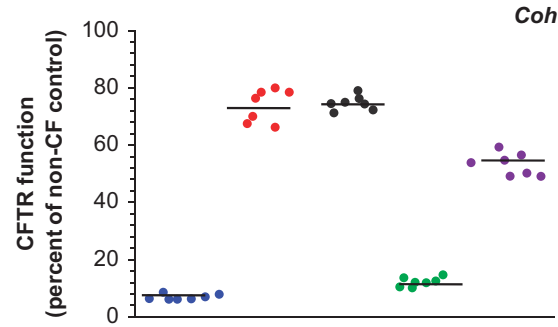

CFTR band C protein
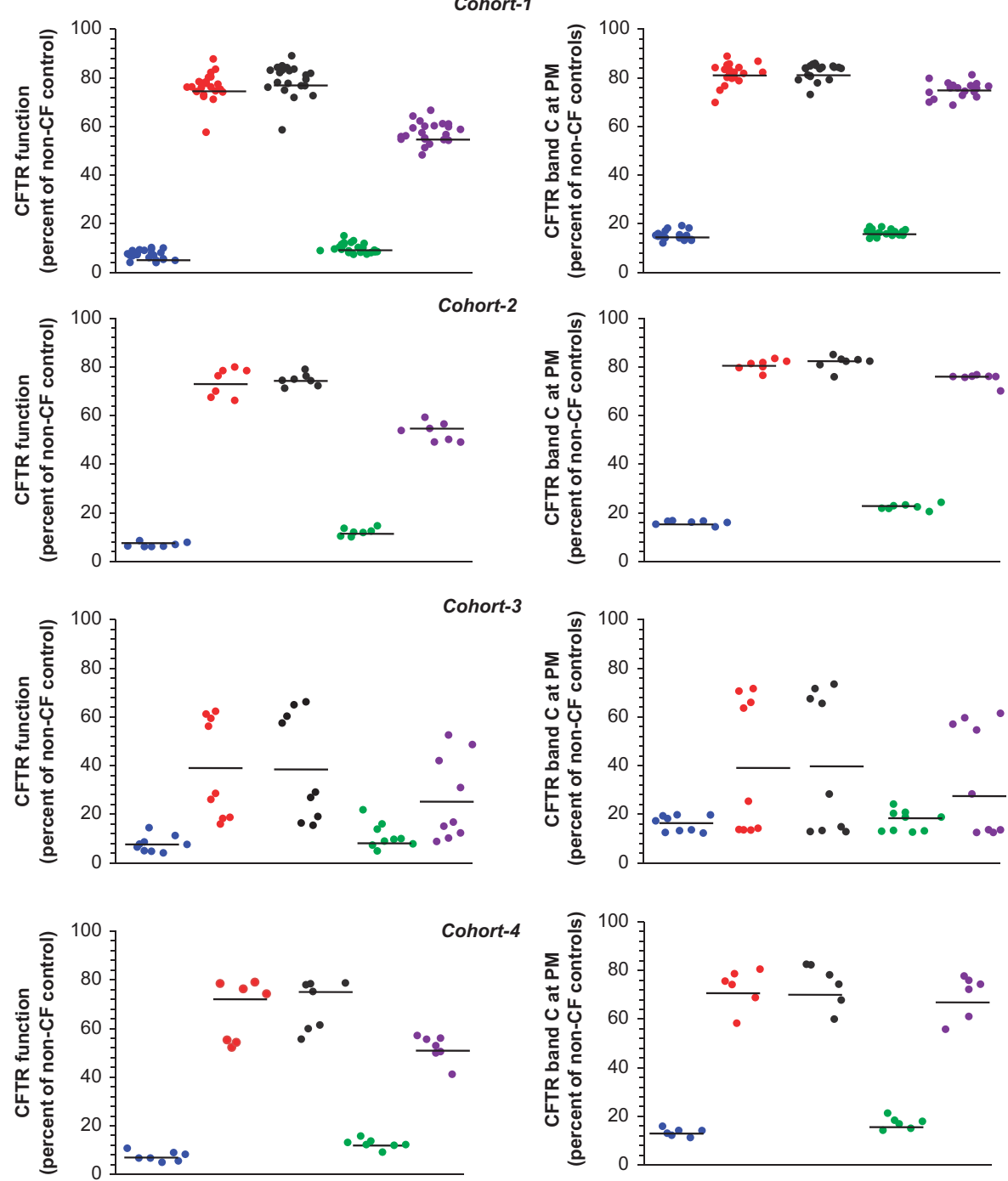

Figure 4 In vitro test of patients responsiveness to treatment in nasal cells freshly collected at week 0 from patients enrolled in the clinical study. (a) Analysis of CFTR function and (b) residual CFTR protein at PM in nasal epithelial cells from 45/52 CF subjects enrolled in the clinical study at baseline and cultured for $18 \mathrm{~h}$ with or without cysteamine $(250 \mu \mathrm{M})$ or with cysteamine plus EGCG $(80 \mu \mathrm{M})$ and then kept for $48 \mathrm{~h}$ in medium or medium added with EGCG. Individual values of patients in different cohorts. (a) Rate of halide efflux, in CF subject of different cohorts expressed as percent of values of five non-CF healthy controls (considered as $100 \%$ of value). The analysis was performed on at least 50 cells per sample and per experiment. (b) Individual values of residual CFTR protein at the PM of CF patients in different cohorts. The values are expressed as percent of five non-CF healthy control. Mean values of independent experiments. Black line represents the mean value. Cys, cysteamine; EGCG, epigallocatechin gallate; Wo, washout. Cys+EGCG, treatment with cysteamine and EGCG; Cys+EGCG+Wo, treatment with cysteamine and EGCG followed by $48 \mathrm{~h}$ of culture with medium alone (washout of both cysteamine and EGCG); Cys+Wo+EGCG, treatment with cysteamine and EGCG followed by $48 \mathrm{~h}$ of cysteamine washout in the presence of EGCG

composite primary end point criteria throughout the study (Fisher's test $P<0.001$ versus treated subjects). Only three responders (all bearing F508del) met the criteria after the first 8 weeks of treatment with cysteamine alone (Fisher's test $P=0.5$ versus untreated patients). Autophagy in nasal cells was restored in all responders after combination treatment but in none of untreated participants throughout the study (Fisher test $P<0.001$ ).

In 23 of 26 responders, at least two of either nasal or sputum CXCL8 and/or TNF- $a$ levels decreased by $>30 \%$ from baseline after combination treatment. None of untreated subjects manifested a similar decrease versus baseline (Fisher's test, $P<0.01$ versus treated subjects) (Figure $6 \mathrm{~d}$ and e).

In 13 out of 34 treated patients (but in none of the untreated patients, Fisher's test $P=0.021$ ) the increase in $\mathrm{ppFEV}_{1}>4$ (relative to baseline) achieved at the end of the combination treatment, was conserved at the post-treatment visit (Table 1). Ten of them belong to the 26 responders, and other 2 (no. 12 and 27, Table 1) met all the sequential steps of responsiveness, except for the sweat test, i.e., restoration of functional CFTR protein and autophagy coupled to stable 
a

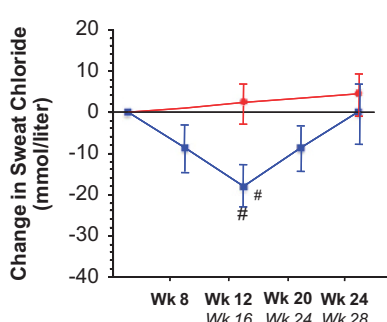

$\begin{array}{lllll}\text { Wk } 8 & \text { Wk } 12 & \text { Wk } 20 & \text { Wk } 24 \\ \text { Wk } 16 & \text { Wk } 24 & \text { Wk } 28\end{array}$

C

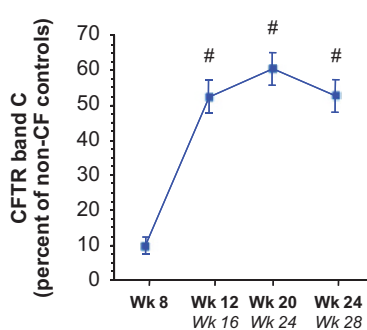

b

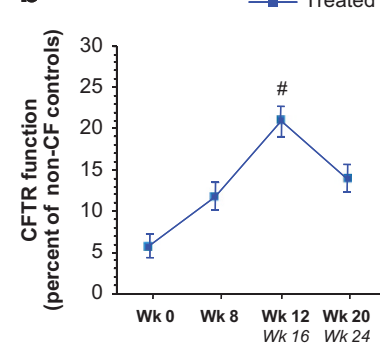

$\begin{array}{ll}\text { Wk } 12 & \text { Wk } 20 \\ \text { Wk } 16 & \text { Wk } 24\end{array}$

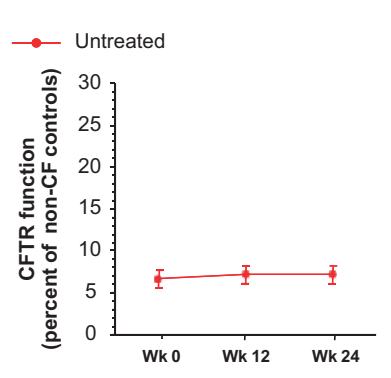

Individual values

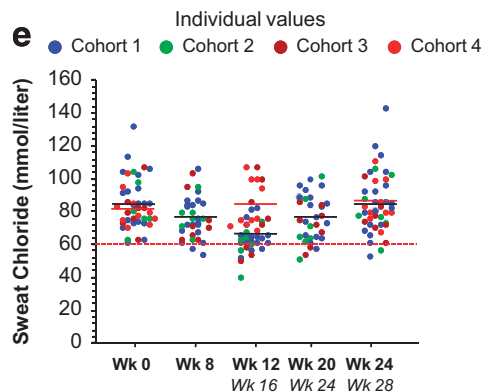

Figure 5 Treatment efficacy on primary end point in study population. (a) Mean change in sweat chloride concentrations (mmol/liter) from baseline during the whole study period in treated (blue line) (weeks 8, 12, 20, 24 of treatment schedule) and untreated (red line) groups (week 12, 24 of observation). In treated cohorts, weeks 16, 24 and 28 (italic) indicate the corresponding time points in the subgroup of patients who received combination treatment for 8 weeks. Data from all cohorts of treated patients at the end of each treatment period are pooled for the analysis. Bars are $95 \% \mathrm{Cl}$. ${ }^{\#} P<0.0001$. (b-c) Mean values of CFTR function (b) and CFTR band C protein (c) expressed as percent of non-CF control subjects in treated (left) and untreated (right) patients during the whole study period. Bars are $95 \% \mathrm{Cl}$. ${ }^{\sharp} P<0.001$ versus baseline. (d) Scatter plot of nasal and sweat measure of CFTR function. The red line represents the estimated linear regression model, with a regression coefficient of -1.1 and its associated $P$-value of 0.0046 . Wk = week. (e) Individual sweat chloride values (mmol/liter) in all study cohorts. Black lines indicate mean values in different cohorts of treated patients (weeks $0,8,12,20,24$ of treatment schedule). Red lines indicate mean values of untreated patients (cohort 4) (week 0, 12, 24 of observation). Red dashed lines indicate the pathological sweat test cutoff value

decrease of inflammatory biomarkers. Six of them (No. 1, 2, $5,8,9,10$ of Table 1) received combination treatment for 8 instead of 4 weeks (subgroup $A$ of cohort 1). This is consistent with preclinical data in mice demonstrating that the beneficial effects of treatment are maintained for several weeks after drug withdrawal. Notably, the occasional (at one single visit) changes in $\mathrm{ppFEV}_{1}$ in untreated cohort were never coupled to a decrease of inflammatory cytokines nor to restoration of CFTR function or autophagy (Table 1). To look at causal relationship between improvement in ppFEV $\mathrm{V}_{1}$ at the end of combination treatment and at the last post-treatment visit, a linear regression model was inferred. A positive regression coefficient was obtained $(0.8)$ with an associated $P=0.000135$.

Predictive value of biomarkers. Personalized approaches to treatment require biomarkers as either (i) predictors of putative individual responsiveness before treatment or (ii) early detectors of efficacy during treatment before clinical benefits become evident.

Analysis of biomarkers in vitro before treatment: In all but one treated patients, in vitro challenge of nasal cells at week 0 with cysteamine plus EGCG restored both CFTR function and band $C$ protein in PM fractions up to $>50 \%$ of non-CF controls. All responders to the treatment in vivo manifested a rescue to $>50 \%$ of CFTR function and protein in vitro (sensitivity: $100 \%$ ), whereas $25 / 29$ (86.2\%) patients who responded in this way in vitro were also responders in vivo.
Thus, rescuing $>50 \%$ of functional CFTR protein in vitro could support the clinical decision to treat in the future with a negative predictive value of $100 \%$ and a positive predictive value of $86.2 \%$ (sensitivity $100 \%$, specificity $50 \%$ ). Therefore, a biomarker for treatment response in vivo could include absolute changes in CFTR protein levels (Supplementary Figure S8, see ROC curve).

Analysis of biomarkers during in vivo treatment: The restoration of CFTR function, as measured ex vivo during in vivo treatment, could be a useful tool to monitor the therapeutic responsiveness before stable beneficial effects on lung inflammation or clinical parameters become evident. The restoration of CFTR function was present in 12/13 (99.3\%) patients who manifested a stable control of inflammation coupled to an absolute increase of ppFEV1 $>4$ percentage points that is maintained at the end of the study.

\section{Discussion}

Our results challenge the currently accepted model that CF is a mere channelopathy that must be treated with agents directly acting on CFTR. The data provide the first evidence that targeting the abnormal proteostasis network results in CFTR repair coupled to clinical benefit in CF patients bearing at least one copy of class II CFTR mutation, including F508del, whereas it is not effective in subjects carrying two class I mutations. This effect can be obtained by two proteostasis regulators, the repurposed drug cysteamine, and the safe 

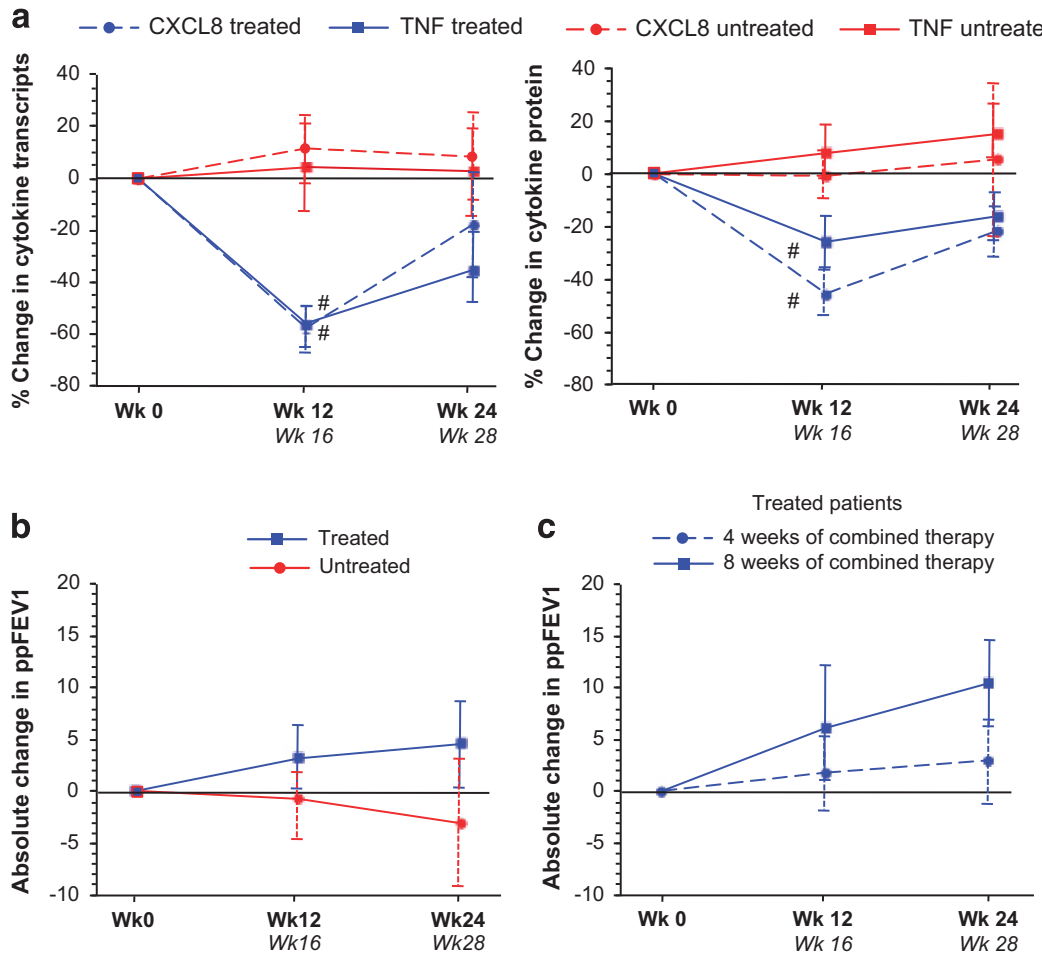
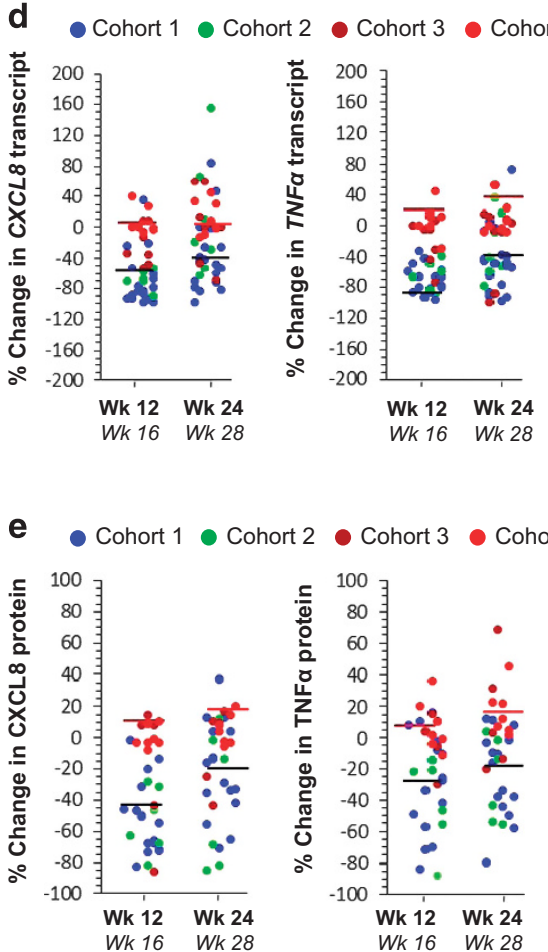

Figure 6 Treatment efficacy on secondary outcomes in study population. (a) Percentage change in CXCL8 and TNF- $\alpha$ transcripts in nasal cells (left) and CXCL8 and TNF- $\alpha$ protein levels in the sputum (right) throughout the study in all treated (blue lines) (weeks 12, 24 of treatment schedule) and untreated (red lines) cohorts (week 12, 24 of

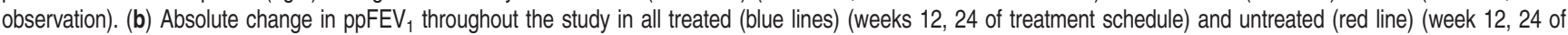
observation) cohorts. (c) Absolute change in $\mathrm{ppFEV}_{1}$ throughout the study in treated patients who received 4 (dashed line) or 8 (solid line) weeks of combination of cysteamine plus EGCG. Bars are $95 \% \mathrm{Cl}$. Wk, week. Week 12 and 24 or Week 16 and 28 (italic) indicate the corresponding time points in patients who received the combination treatment for 4 or 8 weeks, respectively. (d and e) Individual value of percentage change from baseline of CXCL8 (left) and TNF- $\alpha$ (right) transcript levels in brushed nasal cells (top) and CXCL8 and TNF- $\alpha$ protein levels in the sputum (bottom) throughout the study. Black lines indicate mean values of treated patients; red lines indicate mean values of untreated patients. In treated cohorts, Week 12 and 24 or Week 16 and 28 (italic) indicate the corresponding time points in the subgroup of patients who received combination treatment for 4 or 8 weeks, respectively

nutraceutical EGCG, which target two key nodes of mismanaged proteostasis in CF, disabled autophagy and CK2 overactivation.

The effects of treatment are consistent with our mechanistic animal data showing that this combination treatment is not effective in Cftr-null mice (no protein rescuable), whereas it is similarly effective in mice bearing one or two copies of F508del-CFTR, provided that F508del-CFTR mice are autophagy-competent (no effect in Cftr 5 508del/F508del/Becn $1^{+/-}$ mice). In addition, cell-based pre-treatment data in patient's primary nasal cells support the use of combination treatment in patients bearing other severe class II mutations, including N1303K or G85E.

The primary efficacy end point of our clinical study was the restoration of CFTR function in two organs (airways and sweat duct). A short-term treatment with cysteamine plus EGCG, but not with either alone, decreased baseline sweat chloride concentrations by $\sim 20 \%$ while restoring functional CFTR protein up to $\sim 20 \%$ of non-CF controls in nasal cells. The combination treatment reversed the pathogenic cascade of disease as it re-established autophagy in nasal cells, indicating that drugs act 'on-target', in agreement with the hypothesis underpinning our drug design. The restoration of a functional CFTR protein is clinically relevant, as it significantly reduces inflammation markers and increases $\mathrm{FEV}_{1}$ predicted from baseline, even beyond the treatment period.

Personalized medicine is in vogue ${ }^{27-30}$ and CF is a paradigm of heterogeneity in patient response to treatments. ${ }^{25}$ Pooled analysis in large-scale trials might not consider such variability, thus entailing the need of patientcentered approaches to assess drug efficacy. Testing patient's responsiveness to treatment using surrogate markers may help to predict individual responsiveness at the clinical level. In total, $76.5 \%$ of treated patients were responders to the combination treatment, whereas none of untreated participants met these criteria throughout the study. The genotype determined the likelihood of response as all responders carried at least one copy of F508del-CFTR or other class II mutations, but none of them carried two class I CFTR mutants.

$\mathrm{CF}$ is characterized by unresolved inflammation. ${ }^{31}$ After the combination treatment, $88.4 \%$ of responders showed $>30 \%$ decrease of inflammatory cytokines that was retained at the last post-treatment visit, in agreement with mouse data. The prolonged control of lung inflammation may pave the way for the effect of treatment on lung function. Six of 10 patients who received the combination treatment for 8 weeks, retained major clinical benefit until the last post-treatment visit, 

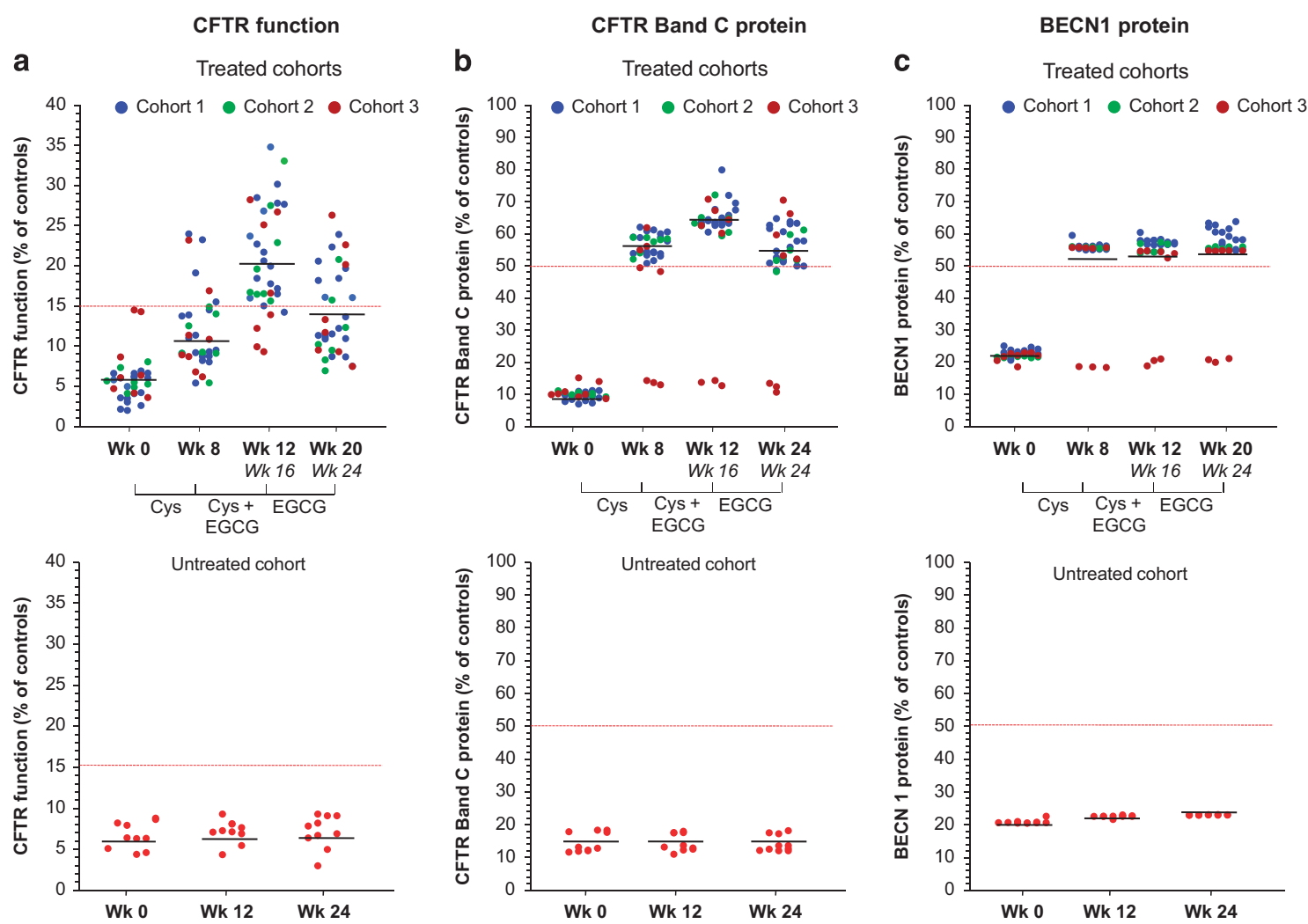

Figure 7 Individual values of biomarkers measured in brushed nasal cells in all study cohorts throughout the study: CFTR function (a) CFTR band C protein (b) and BECN1 (c). Values are expressed as percentage of non-CF controls (considered as 100\% value). Black lines indicate mean values in different cohorts. Red dashed lines indicate $15 \%$ (a) and 50\% (b and c) of values of non-CF control subjects. Wk, week; Cys, cysteamine; EGCG, epigallocatechin gallate. In treated cohorts Wk 12-20 and Wk 16-24 (italic) indicate the corresponding time points in patients who received the combination treatment for 4 or 8 weeks, respectively. In untreated cohort Wk 12 and Wk 24 indicate the corresponding observational time points throughout the study

suggesting a clinical benefit increasing over time within a stably un-inflamed environment.

Testing putative individual responsiveness to treatment by appropriate biomarkers before in vivo therapy should support the decision to treat. We show that restoring CFTR function in vitro in nasal cells in response to cysteamine plus EGCG is highly predictive of whether the combination treatment will restore CFTR function in vivo. Biomarkers of autophagy may add value to monitoring treatment efficacy in patients, according to the supposed mechanism of action. Hence, this in vitro assay may constitute a tool to guide the clinical development of CF treatments, allowing to identify patients who may profit from therapeutic options as the one that we detail here.

Our data indicate sweat test as feasible surrogate biomarker of CFTR function, provided that standardized procedures are respected to reduce intra-individual variability. Sweat chloride changes exhibit significant inverse correlation with CFTR function in nasal cells. Although more variable than CFTR measurements in nasal cells, sweat test should be recommended in clinical trials aimed at CFTR repair.

Our novel therapy results from long-term academic research aiming at refining disease-relevant targets for the action of safe/repurposed drugs. Our combination has another major advantage over existing channel-centered therapies for
F508del-CFTR as it is well tolerated and affordable, potentially costing $>10$-fold less than currently approved drugs. Thus, our affordable safe drug-repurposing strategy will likely set the bar for future clinical trials aiming at repairing CFTR defect.

Our study has some limits mainly related to the small sample size. To compensate for the small number, we selected objective stringent biomarkers of efficacy that likely would not be affected by randomization. The short duration of treatment does not allow to determine the long-term clinical benefit, although patients who had a persistent better clinical benefit had received a longer period of combination treatment. Further placebo-controlled trials on a larger population sample will be required to assess the long-term effects of this combination therapy, especially with respect to pulmonary function, physical and cognitive performance and subjective well being.

\section{Materials and methods \\ Mouse studies}

Mice and treatments: CF mice homozygous for the F508del-CFTR in the FVB/129 outbred background (Cftr ${ }^{\text {m1EUR }}$, F508del, FVB/129, abbreviated

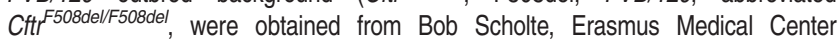
Rotterdam, The Netherlands, CF coordinated action program EU FP6 LSHMCT-2005-018932). ${ }^{32}$ Transgenic KO Cftr mice (B6.129P2-KO Cftrtm1UNC, abbreviated $\mathrm{Cftr}^{-1-}$ ), were purchased from The Jackson Laboratory (Bar Harbor, Maine, USA).33 


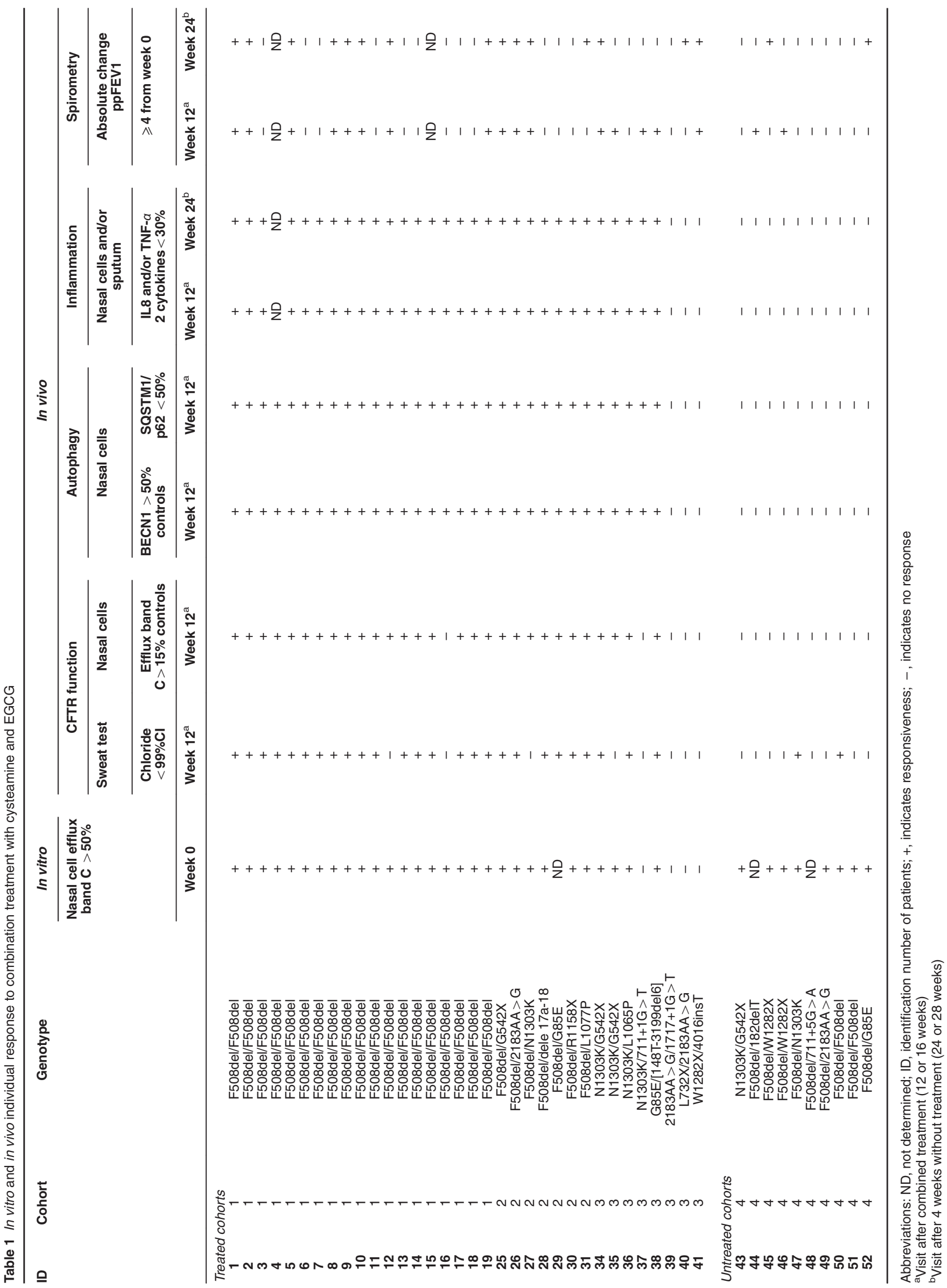


The heterozygous $\mathrm{Cftr}^{\mathrm{F} 508 \mathrm{del} /+}$ males were backcrossed with the heterozygous $\mathrm{Cftr}^{+/-}$females to obtain F508del/null CFTR heterozygous mice (abbreviated $\mathrm{Cftr}^{\mathrm{F} 508 \mathrm{del} /-}$ ). Cftr ${ }^{508 \mathrm{del} /+}$ female mice were backcrossed to the C57BL/6J background Becn1 $1^{+-}$male mice (generous gift from Beth Levine, Center for Autophagy Research, Department of Internal Medicine, UT Southwestern Medical Center, Dallas, USA and Francesco Cecconi, University of Tor Vergata, Rome, Italy) ${ }^{34}$ to obtain at the first generation Becn1 haploinsufficient F508del heterozygous mice (abbreviated $\mathrm{Cftr}^{-508 \mathrm{del} /+} / \mathrm{BeCn}^{+/-}$). These mice $\mathrm{CftF}^{\mathrm{F} 508 \mathrm{del} /}$ ${ }^{+} / B^{2} n 1^{+/}$- were crossbred to obtain Becn1 haploinsufficient F508del homozygous mice (abbreviated $\mathrm{Cftr}^{F 508 \mathrm{del} / \mathrm{F5} 58 \mathrm{del} / \mathrm{BeCn}^{+/-}}$). Mice for the study were aged 8-week-old.

Mice were gavaged with vehicle or cysteamine ( $60 \mu \mathrm{g} / \mathrm{kg}$ in $100 \mu \mathrm{l}$ saline/day) or EGCG (150 $\mu \mathrm{g} / \mathrm{kg}$ in $100 \mu \mathrm{l}$ saline/day) alone for 5 days or with cysteamine plus EGCG for 5 days followed by EGCG alone or vehicle for further 2 weeks, as reported. ${ }^{20}$

At the end of the treatment, mice were anesthetized with Avertine (tribromoethanol, $250 \mathrm{mg} / \mathrm{kg}$, Sigma Aldrich, Milan, Italy, T48402) and a segment of tail was collected for genotyping. Mice were then killed and lungs and intestines collected for analysis. All the procedures in mice were approved by the local Ethics Committee for Animal Welfare (IACUC No. 553 and 582) and were carried out in strict respect of European and National regulations.

Genotyping of the new mouse models: The newly generated $\mathrm{Cftr}^{5508 \mathrm{del} /-}$

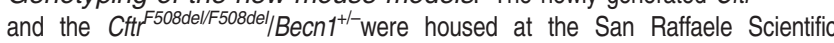
Institute SOPF animal house (Milan, Italy). These mice were provided with a special food, consisting of an equal mixture of SRM-A (Arie Blok, Woerden, The Netherlands) and Teklad 2019 (Harlan Laboratories, San Pietro al Natisone, Udine, Italy) and water acidified to $\mathrm{pH} 2.0$ with $\mathrm{HCl}$ and containing $60 \mathrm{~g} / \mathrm{PEG} 3350,1.46 \mathrm{~g} /$ $\mathrm{NaCl}, 0.745 \mathrm{~g} / \mathrm{KCl}, 1.68 \mathrm{~g} / \mathrm{NaHCO}$ and $5.68 \mathrm{~g} / / \mathrm{Na}_{2} \mathrm{SO}_{4}$. Newborn mice were genotyped by cutting a small piece of tail 12 days after birth. DNA was extracted by digesting tails with Direct PCR Lysis Reagent (Viagen, CA, USA) and $1 \mathrm{mg} / \mathrm{ml}$ Proteinase K overnight at $56^{\circ} \mathrm{C}$. For $\mathrm{Cftr}{ }^{\mathrm{F}} 508 \mathrm{del} / \mathrm{-}$ two PCR reactions were performed. For the $\mathrm{CAtr}^{F 508 d e l / F 508 \mathrm{del}}$ mutation, thermocycling consisted of an initial polymerase activation step at $95{ }^{\circ} \mathrm{C}$ for $5 \mathrm{~min}$, amplification was performed with 30 cycles of $95^{\circ} \mathrm{C}$ for $1 \mathrm{~min}, 52^{\circ} \mathrm{C}$ for $1 \mathrm{~min}$ and $72{ }^{\circ} \mathrm{C}$ for $1 \mathrm{~min}$ with a final extension at $72{ }^{\circ} \mathrm{C}$ for $2 \mathrm{~min}$; for the $\mathrm{Cftr}^{-{ }^{-1}}$ mutation thermocycling consisted of an initial polymerase activation step at $94^{\circ} \mathrm{C}$ for $3 \mathrm{~min}$, amplification was performed with 30 cycles of $94^{\circ} \mathrm{C}$ for $30 \mathrm{~s}$, $57^{\circ} \mathrm{C}$ for $30 \mathrm{~s}$ and $72{ }^{\circ} \mathrm{C}$ for $30 \mathrm{~s}$ with a final extension at $72{ }^{\circ} \mathrm{C}$ for $10 \mathrm{~min}$. For

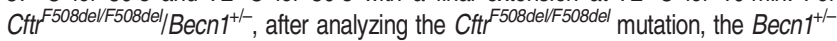
thermocycling consisted of an initial polymerase activation step at $95^{\circ} \mathrm{C}$ for 4 min, amplification was performed with 30 cycles of $95^{\circ} \mathrm{C}$ for $1 \mathrm{~min}, 62^{\circ} \mathrm{C}$ for $1 \mathrm{~min}$ and $72{ }^{\circ} \mathrm{C}$ for 1.30 min with a final extension at $72{ }^{\circ} \mathrm{C}$ for $10 \mathrm{~min}$. The sequences of PCR primers are reported in Supplementary Table S15.

Procedures

Measurement of RPD. RPD was measured as described. ${ }^{20}$ In brief, RPD was measured with a protocol similar to that for nasal potential difference measurements in humans, as described. ${ }^{35,36}$ Mice were anesthetized with Avertine $(250 \mathrm{mg} / \mathrm{kg})$. RPD was sensed with a digital volthometer inserted for $\sim 2 \mathrm{~cm}$ in the rectum. Potentials were measured with respect to a subcutaneous $1 \mathrm{M} \mathrm{NaCl-filled} \mathrm{needle.} \mathrm{A}$ second rectal tube was used for continuous perfusion of drug administration into the rectum. The $\mathrm{Cl}^{-}$containing solution had the following composition: $145 \mathrm{mM} \mathrm{NaCl}$, $4 \mathrm{mM} \mathrm{KCl}, 2 \mathrm{mM} \mathrm{CaCl}_{2}, 1 \mathrm{mM} \mathrm{MgCl}_{2}, 10 \mathrm{mM}$ HEPES, $0.1 \mathrm{mM}$ amiloride, at a final $\mathrm{pH}$ 7.4. The $\mathrm{Cl}^{-}$-free solution had the following composition: $145 \mathrm{mM}$ sodium gluconate, $4 \mathrm{mM}$ potassium gluconate, $4 \mathrm{mM}$ calcium gluconate, $1 \mathrm{mM}$ magnesium gluconate, $10 \mathrm{mM}$ HEPES, $0.1 \mathrm{mM}$ amiloride, at a final pH 7.4. To assess CFTR function in vivo, we evaluated the response of the RPD to the presence of forskolin and luminal amiloride (100 $\mu \mathrm{M}$, to block $\mathrm{Na}^{+}$-dependent RPD) in $\mathrm{Cl}^{-}$-free solutions, as previously described. ${ }^{20}$

Ussing chamber. Chambers for mounting tissue biopsy were obtained from Physiologic Instruments (model P2300, San Diego, CA, USA). Chamber solution was buffered by bubbling with a mixture of $95 \% \mathrm{O}_{2}$ and $5 \% \mathrm{CO}_{2}$. Tissues were short circuited using $\mathrm{Ag} / \mathrm{AgCl}$ agar electrodes. Short-circuit current and resistance were acquired or calculated using the VCC-600 transepithelial clamp from Physiologic Instruments and the Acquire \&Analyze2.3 software for data acquisition (Physiologic Instruments), as previously described. ${ }^{37,38} \mathrm{~A}$ basolateral-to-apical chloride gradient was established by replacing $\mathrm{NaCl}$ with $\mathrm{Na}$-gluconate in the apical (luminal) compartment to create a driving force for CFTR-dependent $\mathrm{Cl}^{-}$secretion. CFTR channels present at the apical surface of the epithelium (lumen side of the tissue) were activated.
Real time and reverse transcript PCR analysis. The analysis was performed as previously described. ${ }^{20,39,40}$ The specifications of primers are reported in Supplementary Table S15.

Immunoblot analysis. Western blot analysis was performed as previously described $^{16,20,21,22}$ with antibodies against the following proteins: BECN1, CFTR, $\beta$-actin, SQSTM1/p62. The densitometric analysis was performed by Image $\mathrm{J}$ software and each data point was expressed as the mean \pm S.D. of independent experiments. The antibodies specifications are reported in Supplementary Table S16.

\section{Human studies}

Patients and study design. An open-label phase-2 clinical trial was conducted between 12 March 2014 and 9 January 2015 at the Department of Translational Medical Sciences, Regional Cystic Fibrosis Care Center, University of Naples Federico II. The protocol of this study is an amendment of the study registered with primary registry EU-CT, Eudract Number \#2013-001258- 82 in order to include CF patients bearing different CFTR mutations for the treatment with cysteamine and EGCG. This amended study was approved by the Italian Agency of Drug and independent institutional local Ethics Committee and was done in compliance with the Good Clinical Practice Guidelines, the amended Declaration of Helsinki, and regulatory requirements. Written informed consent was obtained from all patients and/or their legal guardians.

Among 74 eligible patients in regular follow-up at the at the Department of Translational Medical Sciences, Regional Cystic Fibrosis Care Center, University of Naples Federico II, 52 consenting patients were sequentially enrolled.

Inclusion criteria were as follows: (i) diagnosis of CF with sweat chloride $\geqslant 60$ $\mathrm{mmol} / \mathrm{l}$ and two CF causing mutations at CFTR gene molecular analysis; (ii) age $\geqslant 6$ years; (iii) genotype characterized by (a) F508del on both alleles; (b) F508del or another class II mutation on one allele and a severe CFTR mutation with minimal residual CFTR activity (class I or II) on the other allele or (c) class I mutations on both

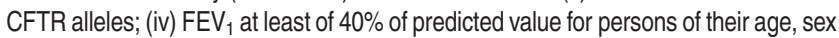
and height.

Exclusion criteria were as follows: (i) treatment with glucocorticoids per os or via inhalation at screening or within 4 weeks before screening visit; (ii) treatment with oxygen; (iii) treatment with other experimental drugs; (iv) referred hypersensitivity (local or general) to cysteamine or penicillamine prior study modifications of therapeutic regimens in patients assuming macrolides, antiasthmatic, mucolytic drugs, Dornase alfa and/or NSAIDs within 28 days before screening visit; (v) organ transplantation; (vi) kidney (creatinine $2 \times$ upper normal limit) or hepatic alterations (ALT and/or AST $5 \times$ upper normal limit) at screening visit; (vii) pregnancy or nursing at screening visit; (viii) refusing to employ contraceptive methods during the study; (ix) psychiatric pathologies or neurological diseases.

After enrollment, safety and efficacy were assessed by clinical and laboratory tests, including nasal brushing, at baseline and at the end of each treatment schedule (Figure 3). Additional assessments were undertaken 4 weeks after drug discontinuation.

We recorded adverse events and clinical and laboratory results throughout the study. Severe adverse events were registered. Pill counts were undertaken at several time points during treatment to evaluate adherence.

Outcomes. Primary end point: (i) sweat chloride values $<15 \%$ from baseline coupled to (ii) CFTR function and CFTR band C protein in brushed nasal epithelial cells $>15 \%$ of non-CF controls.

Secondary end points: (i) restoration of autophagy in nasal cells (BECN1 protein levels $>50 \%$ of non-CF controls and SQSTM1/p62 <50\% from baseline; (ii) reduction from baseline of inflammatory cytokines in both nasal cells and sputum; (iii) absolute change from baseline in the $\mathrm{ppFEV}_{1}$.

Composite criteria to define individual responsiveness to treatment:: Pre-specified criteria that must be simultaneously fulfilled to define individual responsiveness to treatment:

(1) sweat chloride concentrations to values $<-99 \% \mathrm{Cl}$ from the mean of the individual values measured within the preceding 24 months and including the value at week 0;

(2) band C CFTR protein and CFTR channel function in brushed nasal epithelial cells $>15 \%$ of healthy non-CF control values. 
Schedule of treatment. Active treatment comprised oral cysteamine (cysteamine bitartrate, trade name Cystagon, Orphan Europe) with or without EGCG (trade name Epinerve, SIFI Pharmaceuticals). Initially, Cystagon (900 mg/die for patients $\leqslant 12$ years and $1200 \mathrm{mg} /$ die for patients $>12$ years in four divided doses) was given alone for 8 weeks, followed by a combination of Cystagon (same dose) with Epinerve ( $270 \mathrm{mg}$ once daily) for further 4 weeks (or for 8 weeks in a subgroup of 10 F508del-CFTR homozygous patients) followed by Epinerve alone for additional 8 weeks (Figure 3). Throughout, all subjects continued their pre-study medication.

Therapeutic formulation and dose administration of cysteamine and EGCG: Cysteamine: each hard capsule contains $150 \mathrm{mg}$ fixed of cysteamine (as mercaptamine bitartrate). The other ingredients are microcrystalline cellulose, starch, pregelatined, magnesium stearate/sodium lauryl sulfate, colloidal silicon dioxide, croscarmellose sodium; capsule shells: gelatin, titanium dioxide, black ink on hard capsules. The prescribed dose was taken four times a day, every $6 \mathrm{~h}$, just after or with food

EGCG: each tablet contains $135 \mathrm{mg}$ of EGCG. The other ingredients are microcrystalline cellulose, calcium phosphate dibasic, silicon dioxide, magnesium stearate, mono-diglyceride of fatty acids. The prescribed dose was taken fasting.

Pharmacokinetic analysis. Plasma cysteamine concentrations were measured in six F508del/F508del participants at week 8 before and 2, 4 and $6 \mathrm{~h}$ after a single oral dose of cysteamine (300 mg). The methodologies of the analyses for plasma cysteamine levels are described in Supplementary Materials.

Nasal brushing. Freshly isolated brushed nasal epithelial cells were obtained by nasal brushing, as previously described, ${ }^{20,22}$ from enrolled CF patients and from five non-CF consenting healthy volunteers. Brushes with cells were rapidly transferred in 15-ml sterilized tubes containing RPMI1640 medium (Invitrogen, Carlsbad, CA, USA) with 1\% penicillin-streptomycin (Lonza Group LTD, Basel, Switzerland, $17-602 \mathrm{E})$. The tubes were incubated at $37^{\circ} \mathrm{C}$ for $2 \mathrm{~h}$ on a thermal shaker, to remove all cells from brushes. Cells were centrifuged at $800 \times \mathrm{g}$ (2000 rpm) for $20 \mathrm{~min}$. The supernatant fractions were discarded and the cell pellet treated with $150 \mathrm{ml}$ of trypsin-versene (EDTA) solution (Lonza, 17-161) for $4 \mathrm{~min}$ at $37^{\circ} \mathrm{C}$ to disaggregate possible cell clusters. Cells were placed in CELLC T-25 flasks (Sarstedt Ltd, CS300) with BEGM medium after centrifugation at $800 \times g(2000 \mathrm{rpm})$ for $10 \mathrm{~min}$. Non-specific epithelial cells were removed with daily medium changes. In vitro studies before in vivo treatment. Before in vivo treatment (week 0 ) nasal epithelial cells were freshly collected by nasal brushing from the patients enrolled in the study. Nasal brushing from 45 of 52 patients provided a sufficient number of cells for in vitro evaluation. Nasal cells were cultured in vitro for $18 \mathrm{~h}$ with $250 \mu \mathrm{M}$ cysteamine (Sigma Aldrich, M9768) or $80 \mu \mathrm{M}$ EGCG (Sigma Aldrich, E4143) or cysteamine plus EGCG, and then kept in culture with medium alone up to $48 \mathrm{~h}$, in the presence or absence of EGCG, as described. ${ }^{20}$ We tested the rescue of CFTR function by measuring the rate of halide efflux, and the amount of mature bend C CFTR protein by surface biotinylation and western blot as previously described. $^{20}$

Ex vivo analysis during in vivo treatment. Samples collected at week 0 and at each point through the study were immediately used to assess CFTR function and protein levels, BECN1 and SQSTM1/p62 proteins and cytokine expression. The values were compared with those from five non-CF healthy volunteers. ${ }^{20}$

\section{Procedures}

Halide efflux analysis. The analysis of halide efflux was performed in nasal cells, by the iodide-sensitive fluorescent indicator, SPQ (Molecular Probes/ Invitrogen, Carlsbad, CA, USA, M440), as previously described. ${ }^{20}$ In brief, the cells were incubated for $20 \mathrm{~min}$ at $37^{\circ} \mathrm{C}$ in a humidified chamber with $5 \% \mathrm{CO}_{2}$ with the iodide-sensitive fluorescent indicator SPQ, as described (REF). The SPQloaded cells were mounted on a LSM510 Meta confocal microscope (Zeiss, Milan, Italy) with a $37^{\circ} \mathrm{C}$ heated stage and perfused with iodide buffer for 5-8 min. Changes in CFTR-mediated SPQ fluorescence were observed at the $445 \mathrm{~nm}$ wavelength in response to excitation at $340 \mathrm{~nm}$ during perfusion at $37^{\circ} \mathrm{C}$ in nitrate buffer replaced with $130 \mathrm{mM} \mathrm{NaNO}_{3}$ (Sigma Aldrich, S8170) with $20 \mu \mathrm{M}$ forskolin (Fsk; Sigma Aldrich, F6886) plus $100 \mu \mathrm{M}$ IBMX (Sigma Aldrich, 15879) and fluorescence intensity measured for a further 10-12 min. Signals were recorded at 30-s interval. For each minute the average of the fluorescence intensity was measured from 50 cells for population per coverslip and the peak of halide efflux rate (usually after Fsk plus IBMX adding) of cells was calculated in accordance with the Stern-Volmer relationship. ${ }^{41}$ The rates were calculated using SigmaPlot Version 7.1 for each mean fluorescence trace for each time point generated from the 50 cells examined per population per coverslip. ${ }^{41-45}$

Immunoblot analysis, cell surface biotinylation assay and membrane fractionation. The proteins were obtained from nasal epithelial cells. To detect CFTR protein, a large amount $(120 \mu \mathrm{g})$ of protein was loaded. Western blot was performed as previously described ${ }^{20}$ with antibodies against the following proteins: BECN1, CFTR, $\beta$ actin, SQSTM1/p62. The antibodies specifications are reported in Supplementary Table S16.

Insoluble pellet was obtained by centrifugation at $9000 \times \mathrm{g}$ at $4^{\circ} \mathrm{C}$ for $20 \mathrm{~min}$ after lysis and dissolved 5 times in sample buffer, boiled at $95^{\circ} \mathrm{C}$ for 5 min and resolved on a polyacrylamide gel for western blot analysis of SQSTM1/p62, as described. ${ }^{20}$

The densitometric analysis was performed by Image $\mathrm{J}$ software and each data point was expressed as the mean \pm S.D. of independent experiments.

Cell-surface proteins were biotinylated and the PM fraction was collected to detect mature CFTR band $C$ at the PM. Sulfosuccinimidyl-6-(biotinamido) hexanoate (sulfoNHS-LC-Biotin, Pierce, Rockford, IL, USA, 21335), was dissolved at $1 \mathrm{mg} / \mathrm{ml}$ in PBS (Gibco/Thermo Scientific Milan, Italy, 18912-014, pH 8.2), as described. ${ }^{22}$ After homogenization with a Potter-Elvehjem pestle, the cells were centrifuged at $2300 \times \mathrm{g}$ for $15 \mathrm{~min}$ at $4{ }^{\circ} \mathrm{C}$. Supernatant fractions that contain the cytoplasmic and PM fractions were centrifuged $1 \mathrm{~h}$ at $16000 \times g$ at $4^{\circ} \mathrm{C}$; the pellet that contains the intact membrane, was solubilized in Buffer A ( 20 mM Tris-HCl, pH 7.4, 2 mM EDTA, 20 mM 2-mercaptoethanol, $1 \times$ PMSF, $1 \mu \mathrm{g} / \mathrm{ml}$ inhibitor protease cocktail (Sigma Aldrich, P8340)+1\% Triton X-100 (Sigma Aldrich, X-100-RS) and centrifuged $1 \mathrm{~h}$ at $60000 \times g$ in the ultracentrifuge. The supernatants were representative of PM fraction. Equivalent amounts of protein $(500 \mu \mathrm{g})$ were used for streptavidin-agarose affinity isolation (Pierce, Rockford, IL, USA, 20349). Biotinylated proteins of PM immunoblotted against CFTR or FLOT1. The densitometric analysis was performed by Image $\mathrm{J}$ software and each data point was expressed as the mean \pm S.D. of independent experiments.

Measure of TNF-a and CXCL8 expression levels in nasal brushing. RNA extraction was performed as detailed above. For human samples, thermocycling consisted of an initial polymerase activation step at $95^{\circ} \mathrm{C}$ for $5 \mathrm{~min}$, amplification was performed with 40 cycles of $95^{\circ} \mathrm{C}$ for $15 \mathrm{~s}, 60^{\circ} \mathrm{C}$ for $10 \mathrm{~s}$ and $72{ }^{\circ} \mathrm{C}$ for $20 \mathrm{~s}$ with data acquisition at this stage and the reaction finished by the built in melt curve. Expression levels of genes were normalized to the housekeeping gene GAPDH in the same sample. The specifications of primers are reported in Supplementary Table S15.

Measure of TNF- $a$ and CXCL8 levels by ELISA in sputum. Sputum samples were diluted $1: 2$ with PBS and digested with $1 \mathrm{U} / \mu \mathrm{l}$ of DNAse for $4 \mathrm{~h}$ at $37^{\circ} \mathrm{C}$. The supernatants were collected and stored at $-80^{\circ} \mathrm{C}$ until usage. The samples were then centrifuged at $800 \times g$ for $10 \mathrm{~min}$ and the supernatants used to measure TNF- $\alpha$ and CXCL8 levels by means of standard ELISA kits (R\&D Systems, Minneapolis, MN, USA), according to the manufacturer's instructions, as reported. ${ }^{16,46}$ Samples were read in triplicate at $450 \mathrm{~nm}$ in Microplate Reader (BioRad, Milan, Italy) using Microplate Manager 5.2.1 software. Values were normalized to protein concentration evaluated by Bradford analysis.

Statistical analysis. Categorical variables are presented as proportion (\%) and continuous variables as mean (and S.D.) when normally distributed or median (and IQR) when not.

Mice: All laboratory tests on mice were performed at least in triplicate. Betweengroup comparisons were evaluated by one-way analysis of variance, applied to mean (or median) values of continuous variables; post hoc comparisons were made using Bonferroni correction, when appropriate. We set the level of significance at $P<0.05$. Humans: An ethical committee (including statisticians) validated the plan of the study. Normality of data distribution was tested and the effects of treatment in CF patients on either sweat chloride or nasal CFTR function were analyzed by repeated ANOVA, comparing the means of variables measured at different times of treatment, and during the 4-week follow-up period, as previously described. ${ }^{20}$ For brushed nasal cells all laboratory tests on cells were performed at least in triplicate. According to Šidák correction for multiple comparisons, each comparison ${ }^{47}$ was considered significant if $P$-values (for two-tailed test) were $<1-(1-\alpha)^{1 / n}$, where $a$ is the overall type I error probability and $n$ is the number of comparisons. Values registered at the end of combination treatment, either 4-week or 8-week treatment, with cysteamine plus EGCG were 
pooled for the analysis. The Fisher's test was used to assess the proportion of subjects who fulfilled the primary end point between treated (at the end of the combination treatment) and untreated (during an observational period of the same duration) groups for variation of CFTR function (see the exact definition of primary end point above).

For secondary end points a paired $T$-test was used to evaluate differences between two time points in the same group of patients. We used a Fisher's test to evaluate differences between groups (treated versus untreated, as detailed above) for rescue of autophagy, cytokine values in both nasal brushing and sputum, and absolute change in $\mathrm{ppFEV}_{1}$. For prediction tests and biomarkers, unpaired T-tests were uses to compare two groups of quantitative data, correlation tests (Pearson's and Spearman's) were used to search for relationship between quantitative data, linear model were used to search for causal relationship between quantitative data. ROC curve analysis was applied to analyze potential biomarker for treatment response. Data was processed through EXCEL (versions 2010). All data processing and analyses were carried out with SAS statistical software (version 9.2; SAS Institute, Cary, NC, USA) and R software (version 3.0.2).

\section{Conflict of Interest}

LM, VR and GK are listed as inventors on a patent application (No. 13/895741) owned by the No-profit Foundation European Institute for Research in Cystic Fibrosis, describing the use of cysteamine for the treatment of CF. The authors declare no conflict of interest.

Acknowledgements. We thank the participating patients and their families. We thank Dr. Bob Scholte, Erasmus Medical Center Rotterdam, The Netherlands, who provided Cftr ${ }^{\text {m1EUR }}$ (F508del (FVB/129) mice (European Economic Community European Coordination Action for Research in Cystic Fibrosis program EU FP6 SHMCT-2005-018932), Dr. Beth Levine, Center for Autophagy Research, Department of Internal Medicine, UT Southwestern Medical Center, Dallas, USA and Dr. Francesco Cecconi, University of Tor Vergata, Rome, Italy, who provided C57BL/6J background $\mathrm{Becn}^{+/-}$mice, Dr. Valeria R Villella, Dr. Romina Monzani and Dr. Candida Bonelli, European Institute for Research in Cystic Fibrosis, for technical assistance. This study was supported by The European Institute for Research in Cystic Fibrosis (IERFC) non-profit foundation, Italian Cystic Fibrosis Association (LIFC) and Regional Cystic Fibrosis Associations of Campania, Sicilia, Lazio, Puglia (to Dr. L Maiuri, Dr. Raia), E-Rare (Rescue CFTR preclinic) (to Dr. L Maiuri and Dr. Kroemer); Telethon (\#GGP12128) (to Dr. L Maiuri, Dr. Raia, Dr. MC Maiuri), Agence National de la Recherche (ANR) - Projetsblancs; ANR under the frame of E-Rare-2, the ERA-Net for Research on Rare Diseases; Association pour la recherche sur le cancer (ARC); Cancéropôle Ile-de-France; Institut National du Cancer (INCa); Fondation Bettencourt-Schueller; Fondation de France; Fondation pour la Recherche Médicale (FRM); the European Commission (ArtForce); the European Research Council (ERC); the LabEx Immuno-Oncology; the SIRIC Stratified Oncology Cell DNA Repair and Tumor Immune Elimination (SOCRATE); the SIRIC Cancer Research and Personalized Medicine (CARPEM); and the Paris Alliance of Cancer Research Institutes (PACRI) (all to Dr. Kroemer). The funders of the study had no role in study design, data collection, data analysis, data interpretation or writing of the report.

\section{Author contributions}

VR, LM and GK conceived, designed and analyzed the overall study. VR, LM, GK, AM and AT wrote the manuscript. VR and AT designed and wrote the clinical trial protocol. AT, FDG, AS identified patients, executed the trial procedures, dispensed study drugs, collected data and followed up the study participants, under the supervision of VR. AT and FDG coordinated the collaborative work between clinical and laboratory teams and the biochemical work-up of clinical trial samples under the supervision of VR and LM. AT, FDG and AS obtained patient consent. LS performed sweat test analyses. PB and ADP did spirometric analyses. RG and CAL performed nasal brushings. SE did western blot analysis and assessment of CFTR function in nasal brushings, did in vitro cultures on patients nasal cells. DDS, IS and EF did all preclinical experiments on mice, did cross-breading and genotyping of mice, performed mouse treatments, assessment of CFTR function, protein and cytokine assessment and together with MCM analyzed mice data under the supervision of LM. GDR and SL analyzed the pharmacokinetic profile of cysteamine. MCM, AM, GB, SG contributed to data analysis and discussion. GS did statistical analysis. All authors have seen and approved the final version.

1. Ratjen F, Döring G. Cystic fibrosis. Lancet 2003; 361: 681-689.

2. De Boeck K, Zolin A, Cuppens $H$, Olesen HV, Viviani L. The relative frequency of CFTR mutation classes in European patients with cystic fibrosis. J Cyst Fibros 2014; 13: 403-409.

3. Ramsey BW, Davies J, McElvaney NG, Bell SC, Drevinek P, Griese M et al. VX08-770-102 Study Group. A CFTR potentiator in patients with cystic fibrosis and the G551D mutation. N Engl J Med 2011; 365: 1663-1672.

4. Wainwright CE, Elborn JS, Ramsey BW, Marigowda G, Huang X, Cipolli M et al. TRAFFIC and TRANSPORT Study GroupsLumacaftor-Ivacaftor in patients with cystic fibrosis homozygous for Phe508del CFTR. N Engl J Med 2015; 373: 220-231.

5. Boyle A MP, Bell SC, Konstan MW, McColley SA, Rowe SM, Rietschel E et al. VX09-809102 study group. CFTR corrector (lumacaftor) and a CFTR potentiator (ivacaftor) for treatment of patients with cystic fibrosis who have a phe508del CFTR mutation: a phase 2 randomised controlled trial. Lancet Respir Med 2014; 2: 527-538.

6. Davis PB. Another beginning for cystic fibrosis therapy. N Engl J Med 2015; 373: 274-276.

7. Jones AM, Barry PJ. Lumacaftor/ivacaftor for patients homozygous for Phe508del-CFTR: should we curb our enthusiasm? Thorax 2015; 70: 615-616.

8. Amaral MD, Farinha CM. Rescuing mutant CFTR: a multi-task approach to a better outcome in treating cystic fibrosis. Curr Pharm Des 2013; 19: 3497-3508.

9. Cholon DM, Quinney NL, Fulcher ML, Esther CR Jr, Das J, Dokholyan NV et al. Potentiator ivacaftor abrogates pharmacological correction of $\triangle$ F508 CFTR in cystic fibrosis. Sci TransI Med 2014; 6: 246ra96.

10. Veit G, Avramescu RG, Perdomo D, Phuan PW, Bagdany M, Apaja PM et al. Some gating potentiators, including VX-770, diminish $\triangle$ F508-CFTR functional expression. Sci TransI Med 2014; 6: 246ra97.

11. Accurso FJ, Van Goor F, Zha J, Stone AJ, Dong Q, Ordonez CL et al. Sweat chloride as a biomarker of CFTR activity: proof of concept and ivacaftor clinical trial data. J Cyst Fibros 2014; 13: 139-147.

12. De Boeck K, Kent L, Davies J, Derichs N, Amaral M, Rowe SM et al. European Cystic Fibrosis Society Clinical Trial Network Standardisation CommitteeCFTR biomarkers: time for promotion to surrogate end-point. Eur Respir J 2013; 41: 203-216.

13. Amaral MD. Novel personalized therapies for cystic fibrosis: treating the basic defect in all patients. Intern Med 2015; 277: 155-166.

14. Awatade NT, Uliyakina I, Farinha CM, Clarke LA, Mendes K, Sole A et al. Measurements of functional responses in human primary lung cells as a basis for personalized therapy for cystic fibrosis. EBioMedicine 2014; 2: 147-153.

15. Wang X, Venable J, LaPointe P, Hutt DM, Koulov AV, Coppinger J et al. Hsp90 cochaperone Aha1 downregulation rescues misfolding of CFTR in cystic fibrosis. Cell 2006; 127: 803-815.

16. Luciani A, Villella VR, Esposito S, Brunetti-Pierri N, Medina D, Settembre C et al. Defective CFTR induces aggresome formation and lung inflammation in cystic fibrosis through ROS-mediated autophagy inhibition. Nat Cell Biol 2010; 12: 863-875.

17. Venerando A, Franchin C, Cant N, Cozza G, Pagano MA, Tosoni K et al. Detection of phospho-sites generated by protein kinase CK2 in CFTR: mechanistic aspects of Thr1471 phosphorylation. PloS One 2013; 8: e74232.

18. Gahl WA. Early oral cysteamine therapy for nephropathic cystinosis. Eur J Pediatr 2003; 162: S38-S41.

19. Emma F, Nesterova G, Langman $C$, Labbè A, Cherqui S, Goodyer $P$ et al. Nephropathic cystinosis: an international consensus document. Nephrol Dial Transplant 2014; 29: 87-94.

20. De Stefano D, Villella VR, Esposito S, Tosco A, Sepe A, De Gregorio F et al. Restoration of CFTR function in patients with cystic fibrosis carrying the F508del-CFTR mutation. Autophagy 2014; 10: 2053-2074.

21. Luciani A, Villella VR, Esposito S, Gavina M, Russo I, Silano M et al. Targeting autophagy as a novel strategy for facilitating the therapeutic action of potentiators on DF508 cystic fibrosis transmembrane conductance regulator. Autophagy 2012; 8: 1657-1672.

22. Villella VR, Esposito S, Bruscia EM, Vicinanza M, Cenci S, Guido S et al. Disease-relevant proteostasis regulation of cystic fibrosis transmembrane conductance regulator. Cell Death Differ 2013; 20: 1101-1115.

23. Taylor-Robinson D, Whitehead M, Diderichsen F, Olesen HV, Pressler T, Smyth RL et al. Understanding the natural progression in \%FEV1 decline in patients with cystic fibrosis: a longitudinal study. Thorax 2012; 67: 860-866.

24. DeMarco ML, Dietzen DJ, Brown SM. Sweating the small stuff: Adequacy and accuracy in sweat chloride determination. Clin Biochem 2014; 48: 443-447.

25. Corvol H, Thompson KE, Tabary O, le Rouzic P, Guillot L. Translating the genetics of cystic fibrosis to personalized medicine. Trans/ Res 2015; 168: 40-49.

26. Vanscoy LL, Blackman SM, Collaco JM, Bowers A, Lai T, Naughton K et al. Heritability of lung disease severity in cystic fibrosis. Am J Respir Crit Care Med 2007; 175: 1036-1043.

27. Schork NJ. Personalized medicine: time for one-person trials. Nature 2015; 520: 609-611.

28. Jameson JL, Longo DL. Precision medicine-personalized, problematic, and promising. N Engl J Med 2015; 372: 2229-2234.

29. Bilton D. Personalised medicine in cystic fibrosis must be made affordable. Paediatr Respir Rev 2014; 155: 6-7.

30. Bosch B, De Boeck K. Searching for a cure for cystic fibrosis. A 25-year quest in a nutshell. Eur J Pediatr 2015; 175: 1-8. 
31. Cantin AM, Hartl D, Konstan MW, Chmiel JF. Inflammation in cystic fibrosis lung disease: pathogenesis and therapy. J Cyst Fibros 2015; 14: 419-430.

32. vanDoorninck JH, French PJ, Verbeek E, Peters RH, Morreau H, Bijman J et al. A mouse model for the cystic fibrosis delta F508 mutation. EMBO J 1995; 14: 4403-4411.

33. Snouwaert JN, Brigman KK, Latour AM, Malouf NN, Boucher RC, Smithies 0 et al. An animal model for cystic fibrosis made by gene targeting. Science 1992; 257: 1083-1088.

34. Qu X, Yu J, Bhagat G, Furuya N, Hibshoosh H, Troxel A et al. Promotion of tumorigenesis by heterozygous disruption of the beclin 1 autophagy gene. J Clin Invest 2003; 112 : 1809-1820.

35. Fischer H, Fukuda N, Barbry P, Illek B, Sartori C, Matthay MA. Partial restoration of defective chloride conductance in DF508 CF mice by trimethylamine oxide. Am J Physiol Lung Cell Mol Physiol 2001; 281: L52-L57.

36. Illek $\mathrm{B}$, Fischer $\mathrm{H}$. Flavonoids stimulate $\mathrm{Cl}$ conductance of human airway epithelium in vitro and in vivo. Am J Physiol Lung Cell Mol Physiol 1998; 275: L902-L910.

37. Marchelletta RR, Gareau MG, McCole DF, Okamoto S, Roel E, Klinkenberg R et al. Altered expression and localization of ion transporters contribute to diarrhea in mice with Salmonellainduced enteritis. Gastroenterol 2013; 145: 1358-1368.

38. Gondzik V, Awayda MS. Methods for stable recording of short-circuit current in a $\mathrm{Na}$ +-transporting epithelium. Am J Physiol Cell Physiol 2011; 301: C162-C170.

39. Maiuri L, Luciani A, Giardino I, Raia V, Villella VR, D'Apolito M et al. Tissue transglutaminase activation modulates inflammation in cystic fibrosis via PPAR gamma down-regulation. J Immunol 2008; 180: 7697-7705.

40. Amano H, Yamamoto H, Senba M, Oishi k, Suzuki S, Fukushima K et al. Impairment of endotoxin-induced macrophage inflammatory protein 2 gene expression in alveolar macrophages in streptozotocin-induced diabetes in mice. Infect Immun 2000; 68: 2925-2929.

41. Stern M, Munkonge FM, Caplen NJ, Sorgi F, Huang L, Geddes DM et al. Quantitative fluorescence measurements of chloride secretion in native airway epithelium from $\mathrm{CF}$ and non-CF subjects. Gene Ther 1995; 2: 766-774.
42. Verkman AS, Galietta LJV. Chloride channels as drug targets. Nat Rev Drug Discov 2009; 8 : 153-171.

43. Jayaraman S, Teitler L, Skalski B, Verkman A. Longwavelength iodide-sensitive fluorescent indicators for measurement of functional CFTR expression in cells. Am J Physiol Cell Physiol 1999; 277: C1008-C1018.

44. Munkonge F, Alton EW, Andersson C, Davidson H, Dragomir A, Edelman A et al. Measurement of halide efflux from cultured and primary airway epithelial cells using fluorescence indicators. J Cyst Fibros 2004; 3: 171-176.

45. Mansoura MK, Biwersi J, Ashlock MA, Verkman A. Fluorescent chloride indicators to assess the efficacy of CFTR cDNA delivery. Hum Gene Ther 1999; 10: 861-875.

46. Paine R, Standiford TJ, Dechert RE, Moss M, Martin GS, Rosenberg AL et al. A randomized trial of recombinant human granulocyte-macrophage colony stimulating factor for patients with acute lung injury. Crit Care Med 2012; 40: 90-97.

47. Abdi H. Bonferroni and Sidak corrections for multiple comparison. In: Salkind NJ (eds). Encyclopedia of Measurement and Statistics. Sage: Thousand Oaks, CA, USA, 2007, pp 103-107.

(c) (i) (-) $\odot$ This work is licensed under a Creative Commons Attribution-NonCommercial-NoDerivs 4.0 International License. The images or other third party material in this article are included in the article's Creative Commons license, unless indicated otherwise in the credit line; if the material is not included under the Creative Commons license, users will need to obtain permission from the license holder to reproduce the material. To view a copy of this license, visit http://creativecommons.org/licenses/by-nc-nd/4.0/

Supplementary Information accompanies this paper on Cell Death and Differentiation website (http://www.nature.com/cdd) 